\title{
Der Erwerb der Verbflexion durch thailändische Immigrantinnen in der Schweiz. Eine Bestandsaufnahme*
}

\author{
Korakoch Attaviriyanupap (Bern/Nakhon Pathom)
}

\begin{abstract}
This article focuses on the acquisition of German verb inflection by native speakers of Thai, an isolated language which has no concept of inflection at all. The acquisition process of German verb morphology is analyzed based on all the verb inflectional affixes found in the corpus consisting of spontaneous utterances in Standard German produced by 16 female immigrants living in German-speaking Switzerland. It aims to find out a systematic acquisition order of verb inflectional morphemes and the explanation to this sequence, especially to answer the following four questions: 1) What is the acquisition order of verb inflectional morphemes found in this group of informants 2) Are there any differences between the acquisition of finite and that of non-finite verbs? 3) Are there any differences in the verb morphology acquisition of different types of verbs? 4) Does the acquisition of verb inflection by these informants share more similiarities with the instructed or with the natural acquisition of German as a second language?
\end{abstract}

\section{$1 \quad$ Einleitung}

Da die Morphosyntax des Verbs je nach Sprache ganz unterschiedlich aufgebaut sein kann, bereitet der Erwerb des Verbsystems Schwierigkeiten beim Erlernen einer Zweitsprache. Vor allem wenn Muttersprachler einer isolierenden Sprache das verbale Paradigma einer flektierenden Sprache erlernen müssen, bleibt die richtige Auswahl der verschiedenen Verbformen langfristig ein tückisches Problem. Aufgrund des Fehlens der Flexion in der Muttersprache ist es für sie schwierig, dieses grammatische Konzept in der Zweitsprache wahrzunehmen.

Als eine isolierende Sprache unterscheidet sich das Thailändische in seiner Gesamtstruktur sehr stark vom Deutschen als einer flektierenden Sprache mit großem Formenreichtum und entsprechend flexibler Syntax. Um im Deutschen eine richtige Verbform aus dem Paradigma zu verwenden, müssen sowohl morphologische als auch syntaktische Kriterien berücksichtigt werden. Die meisten Untersuchungen zum Erwerb der Flexionsparadigmen bei Verben konzentrieren sich auf Markierungen der verbalen Kategorien, insbesondere Person, Numerus, Tempus und Aspekt. In Untersuchungen zum Erwerb von Tempus und Aspekt (vgl. z. B. Tmangraksat 1985; von Stutterheim 1986; Dietrich et al. 1995) sollte man sich bei der Analyse jedoch nicht auf die Morphologie beschränken. Die syntaktischen Mittel wie z. B. die richtige Auswahl eines Hilfsverbs oder aber auch die entsprechende Platzierung der finiten und infiniten Verben müssen mit einbezogen werden. Hingegen wird in Untersuchungen, die

\footnotetext{
*Für Kommentare und Vorschläge möchte ich Elke Hentschel, Beat Siebenhaar und einem/r anonymen GutachterIn herzlich bedanken. Klaus Peter danke ich auch sehr für die konstruktiven Diskussionen und die sprachlich-stilistische Verbesserung dieses Beitrags.
} 
sich der Morphologie widmen, meistens nur der Ausdruck der Subjekt-Verb-Kongruenz durch die Personalendung behandelt (vgl. z. B. Köpcke 1987; Blackshire-Belay 1991).

Die vorliegende Arbeit beschränkt sich zwar auf den morphologischen Aspekt, aber nicht nur auf die Personalflexion. Im Mittelpunkt der Untersuchung steht der Gebrauch bzw. das Auftreten aller Flexive im deutschen Verbparadigma, wobei sowohl finite als auch infinite Verbformen behandelt werden. Das Zusammenspiel zwischen den finiten und infiniten Verbteilen wird dagegen nicht berücksichtigt. Ziel der vorliegenden Arbeit ist es, den Erwerbsverlauf der Verbflexive sowie die damit verbundenen Schwierigkeiten festzustellen. Es wird in erster Linie den folgenden Fragen nachgegangen:

- Welche Flexionsmorpheme werden zuerst erworben und welche scheinen für den Erwerb problematisch zu sein? Wie sieht ihre Erwerbssequenz aus?

- Bestehen Unterschiede beim Erwerb der Flexionsmorpheme für finite und infinite Verbformen?

- Wie sieht der Erwerb der Verbflexion bei verschiedenen Verbtypen aus?

- Verläuft der Erwerb der Verbflexion der Informantinnen wie im gesteuerten oder wie im ungesteuerten Zweitspracherwerb? ${ }^{1}$

\section{Zum Korpus}

Als Korpus dienen Transkriptionen der Äußerungen von 16 thailändischen Immigrantinnen, die im Zeitraum zwischen Juni und Oktober 2004 während 10 informeller Gespräche auf Hochdeutsch aufgenommen wurden. Bei jeder Aufnahmesituation waren mindestens drei Personen anwesend: die Informantin, eine Begleitperson, die nicht über Thai-Kenntnisse verfügt, und die Autorin der vorliegenden Arbeit. Die Länge der Transkription ist bei jeder Informantin unterschiedlich, sowohl in Bezug auf die zeitliche Länge als auch die Wortanzahl betreffend. Die kürzeste Transkription umfasst 15 und die längste 40 Minuten. Die Anzahl der Wörter ${ }^{2}$ reicht von 128 bis 2'404.

Alle Informantinnen sind mit Schweizern verheiratet und wohnen in der Region Bern. Ihre Aufenthaltsdauer bis zum Zeitpunkt der Datenerhebung reicht von ca. 7 Monaten bis zu 15 Jahren. Einige von ihnen besuchten zum Erhebungszeitpunkt gerade einen Deutschkurs, während andere diese Phase längst hinter sich hatten, weil sie schon sehr lange in der Schweiz leben. Der Besuch von mindestens einem Deutschkurs als Kriterium bei der Auswahl der Informantinnen hatte einerseits zum Ziel, zu kontrollieren, dass sich die Informantin der

\footnotetext{
1 Der Hochdeutscherwerb bei thailändischen Immigrantinnen in der Schweiz ist m. E. "speziell", denn dieser lässt sich weder dem Deutscherwerb bei ausländischen Studierenden oder Kursteilnehmenden eines Sprachinstituts im klassischen "gesteuerten" Unterricht noch der Situation bei ausländischen Arbeitsimmigranten zuordnen, der den klassischen "ungesteuerten" bzw. "natürlichen" Zweitspracherwerb darstellt. Die diglossiche Situation in der Schweiz führt dazu, dass es für Immigranten kaum möglich ist, Hochdeutsch ungesteuert zu erwerben. Allerdings wird auch ihr Hochdeutschgebrauch eher durch Kontakt mit Deutschschweizern in natürlicher Umgebung intensiviert. Es handelt sich um eine Mischform des Zweitspracherwerbs, deshalb ist es interessant der Frage nachzugehen, ob der Zweitspracherwerb dieser Informantinnen mehrheitlich gesteuert oder ungesteuert verläuft.

${ }^{2}$ Es handelt sich hier um automatische Wörterzählung. Als Wortgrenze gilt ein Leerzeichen. Es kam allerdings vor, dass im Laufe der Gespräche nicht nur Deutsch, sondern auch Thailändisch gesprochen wurde. Beim Zählen der Wörter werden thailändische Wörter nur berücksichtigt, wenn sie zu deutschen Äußerungen gehören, d. h. vor allem wenn sie mitten im Satz und in wenigen Fällen am Anfang oder am Ende einer Äußerung stehen, und nicht weggelassen werden können. Pausenzeichen, unverständliche Wörter sowie längere Äußerungen auf Thailändisch werden nicht mitgezählt.
} 
Diglossie in der Schweiz bzw. des Unterschieds zwischen den beiden Varianten der deutschen Sprache (Mundart und Hochdeutsch) bewusst ist. Andererseits ergab sich so die Möglichkeit, den Hochdeutscherwerb dieser Informantinnen mit dem im üblichen Deutschunterricht vermittelten Inhalt zu vergleichen, um bei der Analyse zusätzliche Aspekte zu ergänzen. $\mathrm{Ob}$ der Umgebungsdialekt den Hochdeutscherwerb im Bereich der Verbflexion beeinflusst, spielt für die hier vorgenommene Untersuchung keine weitere Rolle. Es sei an dieser Stelle jedoch darauf hingewiesen, dass ein solcher Einfluss bei näherer Betrachtung aller aufgetretenen Flexive nicht ganz ausgeschlossen werden kann.

Die im Folgenden zu analysierenden Verbformen betreffen nur die deutschen Verben. ${ }^{3}$ Die Anzahl der analysierten Verbformen (tokens) bei jeder einzelnen Informantin liegt zwischen 30 und 351. Insgesamt gibt es im Korpus 153 Lexeme (types). Auch das Repertoire der types ist bei jeder einzelnen Informantin unterschiedlich groß, nämlich zwischen 17 und 58 . Die Mehrheit der Lexeme kommt nur vereinzelt vor, d. h. nur eine oder höchstens einige Informantinnen teilen dieselben Lexeme. Die Verben, die bei allen 16 Informantinnen auftreten, sind sein, machen und sprechen. Ebenfalls von den meisten Informantinnen verwendet werden haben, können, wissen (von jeweils 15 Informantinnen), sagen (von 14 Informantinnen) sowie arbeiten, gehen und kommen (von jeweils 13 Informantinnen). Obgleich diese 10 Verben zufällig vorkommen und bei der Datenerhebung nicht gezielt elizitiert wurden, vertreten sie recht gut funktional unterschiedliche Verbgruppen (Kopula-, Hilfs-, Modal- und Vollverben), die zugleich verschiedene Konjugationstypen repräsentieren. Deshalb soll in 4.4 näher auf die Flexion dieser 10 Verben eingegangen werden.

\section{$3 \quad$ Kategorisierung der Verbformen im Korpus}

Vor der Analyse der verbalen Flexive wurden alle Verbformen bei jeder einzelnen Informantin in eine eigens entwickelte Analysetabelle übertragen. Alle Verbformen wurden in drei Gruppen eingeteilt: unzuordenbare, infinite und finite Verbformen. Unter den infiniten Verbformen gibt es noch zwei Untergruppen: Infinitiv und Partizip Perfekt. Bei den finiten Verbformen wurden die Verben weiter nach Person und Numerus unterteilt. Im Folgenden soll zunächst jede einzelne Kategorie samt den Kriterien, die der Einordnung der analysierten Verbformen zugrunde lagen, beschrieben werden. Es handelt sich hier eindeutig nicht um morphologische Kriterien, wie man sie sonst für das Deutsche zur Unterscheidung von finiten und infiniten Verbformen einerseits und zur Feststellung der Kongruenz zwischen dem Subjekt und dem finiten Verb verwendet, sondern eher um syntaktische, semantische sowie kontextorientierte Kriterien.

\subsection{Unzuordenbare Verbform}

Im Korpus lässt sich ein Teil der Verbformen weder finiten noch infiniten Verbformen zuordnen. Hier sind einige Beispiele dafür:

(1) ... ja wenn neu anfang mit dem kind [SOI-107]

(2) ... PHUT THUENG schwierig ha + fahren in swiss ... [NAH-200]

(3) nein aber mit bei der arbeit tut ${ }^{74}$ schon ... [A-68]

(4) öhö rechnen zu machen [BAS-122]

(5) ja schon vergessen [NIT-128]

\footnotetext{
${ }^{3}$ Im Korpus lassen sich auch englische Verben finden. Nur 4 Informantinnen haben kein einziges englisches Verb verwendet. Der Prozentsatz der deutschen Verben ist allerdings bei den meisten Informantinnen etwa 90\% und mehr, bis auf einen Ausnahmefall, bei dem der Anteil 76.39\% ausmacht.

${ }^{4}$ Das phonetische Zeichen [?] ist ein Hinweis darauf, dass ein Verschlusslaut im Auslaut zwar feststellbar ist, aber bei der Realisierung nicht gelöst wird.
} 
Diese Verbformen wurden als unzuordenbar klassifiziert. Es handelt sich hauptsächlich um folgende Fälle:

- Verbformen in subjektlosen Sätzen: Solche Fälle gehen z. T. auf Satzbrüche bzw. Anakoluthe zurück, könnten m. E. aber auch durch die Muttersprache verursacht oder zumindest begünstigt sein, weil im Thailändischen das Subjekt nicht selten weggelassen wird, wenn es im Kontext erkennbar ist, vor allem wenn es sich um die erste Person ich handelt (mehr dazu in Attaviriyanupap 2004). In vielen Fällen kann man ein semantisches Subjekt feststellen. Zu den unzuordenbaren Verbformen zählen deshalb nur Verben, deren Subjekt unklar ist, während Verben, denen ein Subjekt zugeordnet werden konnte, im Folgenden als finite Verbformen gelten.

- Verbformen, die als das einzige lexikalische Wort in den jeweiligen Äußerungen vorkommen: Sie kommen insbesondere beim Nachfragen oder beim Wiederholen von Äußerungen der anderen Gesprächsteilnehmerin(nen) vor. Solche Verbformen können weder den finiten noch den infiniten Verben zugeordnet werden.

- Verbformen, die evtl. auch einer anderen Wortart zugerechnet werden könnten: Es kann unklar sein, ob ein Wort ein "Substantiv" oder ein "Verb" ist. Es scheint in dem betroffenen Wort jedoch den Ansatz einer Handlung zu geben, vgl. z. B. rechnen in Bsp. (4), so dass sie m. E. als Verb markiert werden sollten. Trotzdem ist es bei solchen Fällen nicht aufschlussreich, die Flexionsanalyse durchzuführen. Sie gehören deshalb zu den unzuordenbaren Verbformen.

- Verbformen, die sowohl semantisch als auch formal entweder Infinitive oder Partizipien sein könnten: Die Verbform vergessen in Bsp. (5) wurde z. B. als unzuordenbar klassifiziert, weil sowohl der Infinitiv als auch das Partizip des Verbs dieselbe Wortform aufweisen und sich die Form ohne Hilfsverb nicht dem Partizip Perfekt zuordnen lässt. ${ }^{5}$

\subsection{Partizip Perfekt}

Hier sind einige Beispiele von Verbformen, die in der Analysetabelle zu den Partizipien gehören:

(6) wenn $\underline{i}$ hochdeutsch gelern und ich hat ${ }^{\urcorner}$gehört $^{7}$ [SOI-161]

(7) i nid gut gemach vom kleidung nein [NI-172]

(8) ... ich habe wein trinke in ah restaurant... [NUCH-25]

(9) ich hab ${ }^{\urcorner}$vergessen zuetue [PSOI-22]

(10) ah ja ja ja verboten [SO-222]

Diese Verbgruppe lässt im Vergleich zu anderen Kategorien mit weniger Aufwand feststellen, weil die Partizip-Morphologie insbesondere formal sehr stark markiert ist. Folgende Kriterien werden zur Feststellung des Partizips Perfekt herangezogen:

- Verbformen mit ge-: Diese eigenständige Silbe, die ein Teil des grammatischen Morphems zur Bildung des Partizips Perfekt ist, lässt sich als ein zuverlässiges Kriterium zur Feststellung dieser infiniten Verbform im Deutschen anwenden. Alle Verbformen, die im Korpus mit ge- als Präfix auftreten, werden als Partizip II

\footnotetext{
5 Ein wichtiger Grund für die Klassifizierung von vergessen als unzuordenbar ist hier außerdem, dass Partizipien bei dieser Informantin sonst immer gemeinsam mit dem Hilfsverb haben auftreten.
} 
klassifiziert, egal ob sie zusammen mit einem Hilfsverb in Erscheinung treten oder nicht und egal ob die entsprechend richtige Endung (-en oder $-t$ ) auftritt bzw. gesprochen wird oder nicht.

Verbformen ohne ge-, die einem Hilfsverb folgen: Es kann sich dabei um richtige oder falsche Verbformen handeln. Da sie ihren Status als Partizip formal nicht eindeutig genug aufweisen, werden sie erst als Partizipien klassifiziert, wenn die betroffene Informantin auch an anderen Stellen mindestens eine "ge-Form" verwendet hat und an dieser Stelle gerade von einer Handlung in der Vergangenheit sprach, vgl. Bsp. (8).

- Verbformen ohne ge-, die zwar keinem Hilfsverb folgen, aber einen Ablaut aufweisen: Diese werden ebenfalls als Partizip Perfekt klassifiziert, wenn der aufgetretene Stammvokal derjenige ist, der im Partizip Perfekt vorkommt wie z. B. verboten, geboren. Bei solchen Verben wird bei der Partizipbildung ohnehin nicht präfigiert.

\subsection{Infinitiv}

Hier sind einige Beispiele für Verbformen, die der Kategorie Infinitiv zugeordnet wurden:

(11) $\quad \ldots$ ich mut m $^{7}$ schaue wie is schweiz [JOOB-29]

(12) du can komm gall? [NI-118]

(13) ich komm to + office vom mann arbeiten [NI-102]

(14) .. dort habe ich nicht zu tun ... [A-43]

(15) ich habe zwei leute zum ah + duschen [BAS-128]

(16) and she helfe mi vom stay hier öh vom schauen mann [NI-16]

(17) ich hab vergessen zuetue [PSOI-22]

(18) bitte mit soi ah + hochdeutsch sprechen [SOI-159]

Im analysierten Korpus findet sich nur Infinitiv Präsens Aktiv, der im Deutschen auch als Nennform gilt. Die Endung -en (bzw. -eln, -ern) ist an sich ein schlechtes formales Kriterium zur Feststellung eines Infinitivs im Deutschen, weil sie wie bei einer flektierenden Sprache üblich multifunktional ist. Sie gilt beispielsweise auch als Personalendung für die 1. und 3. Person Plural sowie für einige Partizipien. Im Korpus ist die Abgrenzung noch komplizierter, weil diese Endung bei den meisten Informantinnen bei fast allen Kategorien auftritt. Die in der vorliegenden Untersuchung als Infinitive kategorisierten Verbformen betreffen deshalb nur Verben in folgenden Fällen:

- nach Modalverben (auch englischen, vgl. Bsp. (12)) und einigen anderen Verben, die einen Infinitiv einleiten können (z. B. gehen, kommen). Es ist aber durchaus möglich, dass die gebrauchte Form nicht richtig ist, z. B. eine falsche Endung hat;

- zusammen mit der sog. Infinitiv-Partikel zu, egal ob sie das betroffene Verb einleitet oder in das Verb integriert wird;

- nach zum, vom oder für, wenn die Wörter eindeutig keine Substantive sind. Der Ansatz eines Verbs ist einerseits durch das prototypische Merkmal "Handlung" erkennbar und die Form ist andererseits durch eine entsprechende richtige Infinitivkonstruktion in der Zielsprache Deutsch - gleichgültig ob ohne $z u$, mit $z u$ oder mit um zu-ersetzbar, vgl. Bsp. (15), hier hätte man die Konstruktion haben $+z u+$ Infinitiv verwendet;

- ähnliche Konstruktion wie ein reiner Infinitiv, der als Ersatzform des Imperativs verwendet werden kann, vgl. Bsp. (18). 


\subsection{Finite Verbformen}

Alle Verbformen, die sich keiner der oben erwähnten Kategorien zuordnen lassen, werden als finite Verben klassifiziert. Es sei jedoch anzumerken, dass hier eigentlich potentielle bzw. "vermutlich" flektierte finite Verbformen gemeint sind. Die Bezeichnung "vermutlich" geht auf die Tatsache zurück, dass man bei der Einordnung noch nicht sagen konnte, ob die Finitheit bei diesen Informantinnen erworben ist bzw. ob die Informantinnen die Verbflexion in Bezug auf die Unterscheidung "finit" vs. "infinit" überhaupt beherrschen oder nicht. Es handelt sich hauptsächlich um Verbformen, die nach den oben beschriebenen Kriterien für infinite Verbformen eindeutig weder Infinitive noch Partizipien sind. Darüber hinaus kann man, zumindest semantisch bzw. mithilfe des Kontexts, Subjekte zu diesen Verbformen feststellen.

\subsection{Verteilung der Verbformen}

Die folgende Abbildung gibt einen Überblick über die Verteilung aller im Korpus gefundenen Verbformen nach den oben erwähnten Kategorien.

Abbildung 1: Verteilung der Verbformen

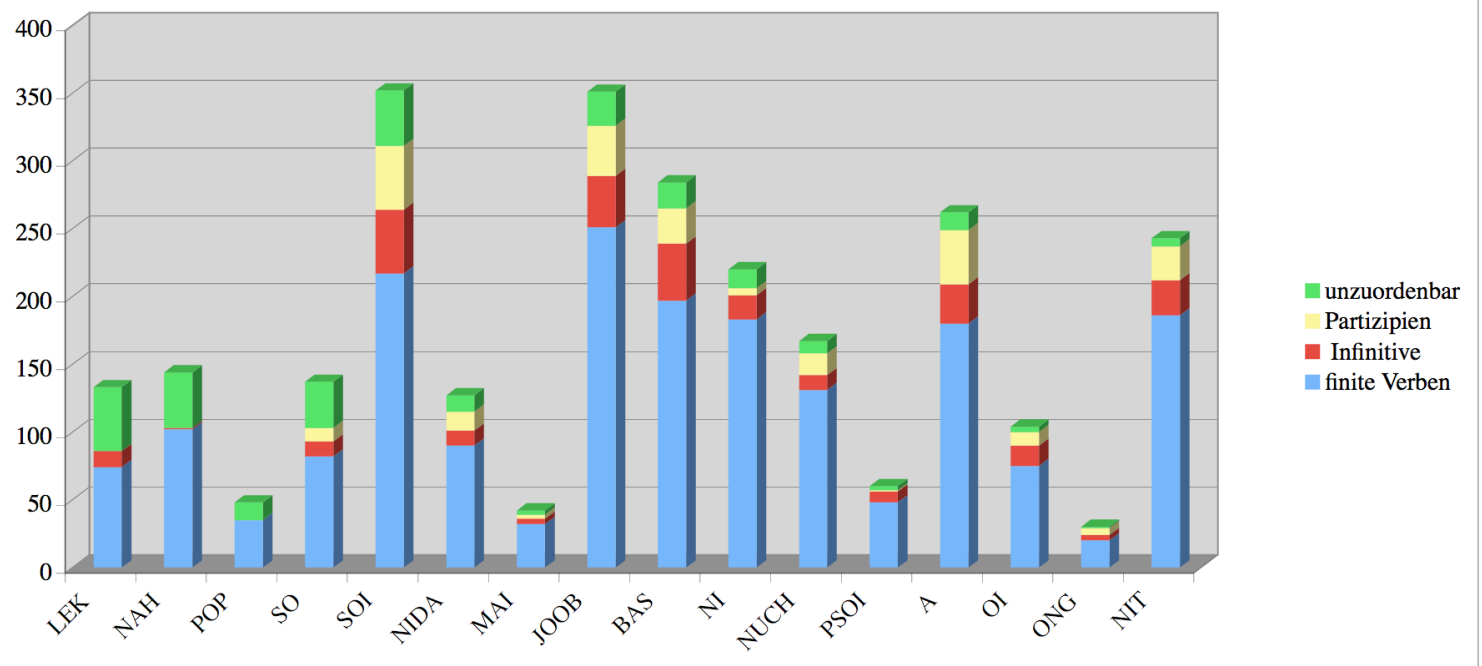

Informantinnen mit einem großen Anteil an unzuordenbaren Verbformen weisen einen niedrigen Erwerbsstand auf. In Abbildung 1 werden die Daten deshalb sortiert, indem die Informantinnen entsprechend dem Anteil an unzuordenbaren Verbformen in ihren Äußerungen absteigend von links nach rechts auf der Achse dargestellt werden. Der Anteil der infiniten Verbformen ist ebenfalls von Bedeutung, weil das Auftreten als infinit klassifizierbarer Verben auf einen höheren Erwerbsstand der betroffenen Informantinnen hinweisen. Bei denjenigen Informantinnen, die überhaupt keine infiniten Verbformen bilden, ist klar geworden, dass sie keine Unterscheidung zwischen finiten und infiniten Verben machen. Bei ihnen ist auch eine geringe Anzahl der verbalen Flexive zu erwarten.

\section{Erwerb der verbalen Flexionsmorpheme}

Bei der Analyse der Flexion, bei der sowohl finite als auch infinite Verbformen berücksichtigt werden, stehen einerseits die Größe der Varianz von gebrauchten Flexionsmorphemen und andererseits die korrekt gebrauchten Verbformen im Mittelpunkt. 


\subsection{Varianten der vorkommenden verbalen Flexionsmorpheme}

Da sich im Korpus Präteritumformen nur bei wenigen Verben (types) finden lassen und deren tokens sehr gering ist, wird bei der Analyse des Erwerbs der Verbflexion das Präteritummorphem nicht getrennt behandelt. Alle finiten Verben im Präsens und Präteritum teilen durch ihre synthetische Formenbildung ohnehin dieselben Personalendungen. Die in der vorliegenden Studie behandelten Flexionsmorpheme der deutschen Verben betreffen deshalb die in Tabelle 1 aufgelisteten Affixe.

Tabelle 1: Distribution der zu untersuchenden Flexive

\begin{tabular}{|l|l|}
\hline$-(e) n$ & $\begin{array}{l}\text { finit: 1. Pers. Pl., 3. Pers. Pl. (z. B. wir gehen, sie gehen) } \\
\text { infinit: Infinitiv, Partizip Perfekt (z. B. vergessen) }\end{array}$ \\
\hline$-\varnothing$ & finit: 1. Pers. Sg., 3. Pers. Sg. (z. B.. ich kann, er kann) \\
\hline$-e$ & finit: 1. Pers. Sg. (z. B. ich gehe) \\
\hline$-(e) t$ & $\begin{array}{l}\text { finit: 3. Pers. Sg., 2. Pers. Pl. (z. B. er geht, ihr geht) } \\
\text { infinit: Partizip Perfekt (z. B. verkauft) }\end{array}$ \\
\hline$-(e) s t$ & finit: 2. Pers. Sg. (z. B. du gehst) \\
\hline ge- +-(e)t & infinit: Partizip Perfekt (z. B. gemacht) \\
\hline ge- +-en & infinit: Partizip Perfekt (z. B. gegangen) \\
\hline
\end{tabular}

In Abbildung 2 werden alle im Korpus aufgetretenen Flexionsmorpheme dargestellt. Es sei zu dieser Darstellung Folgendes anzumerken:

- Das Nullmorphem bzw. das Zeichen - $\emptyset$ markiert eine Verbform, bei der keine Endung angehängt wird. Der Ablaut bzw. die Vokaländerung im Verbstamm wird hier nicht widergespiegelt. Beispielsweise werden sowohl kann als auch könn der Endung - $\varnothing$ zugeordnet.

- Das Flexionsmorphem -(e)st wird in der Abbildung als - $(e) s(t)$ angegeben, weil es im Korpus mehrheitlich nur als $-s$ realisiert wird. ${ }^{6}$

- Das Kopula-/Hilfsverb sein müsste wegen seiner Suppletivformen eigentlich gesondert betrachtet werden. Dies erfolgt jedoch nicht, da die gesonderte Behandlung dieses Verbs das Gesamtbild nicht beeinflussen würde. Mit Ausnahmen von waren (-en) und bist (-st) wurden alle vom Verb sein gebildeten Formen, die als finite Verben klassifiziert worden sind, den endungslosen Formen $(-\varnothing)$ zugeordnet (d. h. also bin, is, isch und war).

Die von den Informantinnen gebrauchten verbalen Flexionsmorpheme verteilen sich entsprechend der folgenden Abbildung. Um das Varianzspektrum der aufgetretenen Flexive bei jeder einzelnen Informantin hervorzuheben, wird die Verteilung der aufgetretenen Flexive nach prozentualen Anteilen dargestellt. Die jeweilige Anzahl von types und tokens werden

6 Die thailändischen Informantinnen haben vor allem Probleme, sowohl einzelne Plosive als auch Konsonantencluster im Auslaut zu artikulieren. Der Laut [s] kommt im Thailändischen nie am Silbenende vor. Solche Schluss-s werden sehr häufig mit dem ungelösten Plosiv [t 7 ] ersetzt. Die Endung - $(e) s t$ wird deshalb kaum realisiert. Falls der Laut [s] einem Verbstamm angehängt wird, wird die Verbform dem Flexionsmorphem $-(e) s(t)$ zugeordnet. 
allerdings angegeben und außerdem von links nach rechts sortiert (types $=1$. Sortierschlüssel, tokens $=2$. Sortierschlüssel).

Abbildung 2: Aufgetretene Flexionsmorpheme bei allen Verben

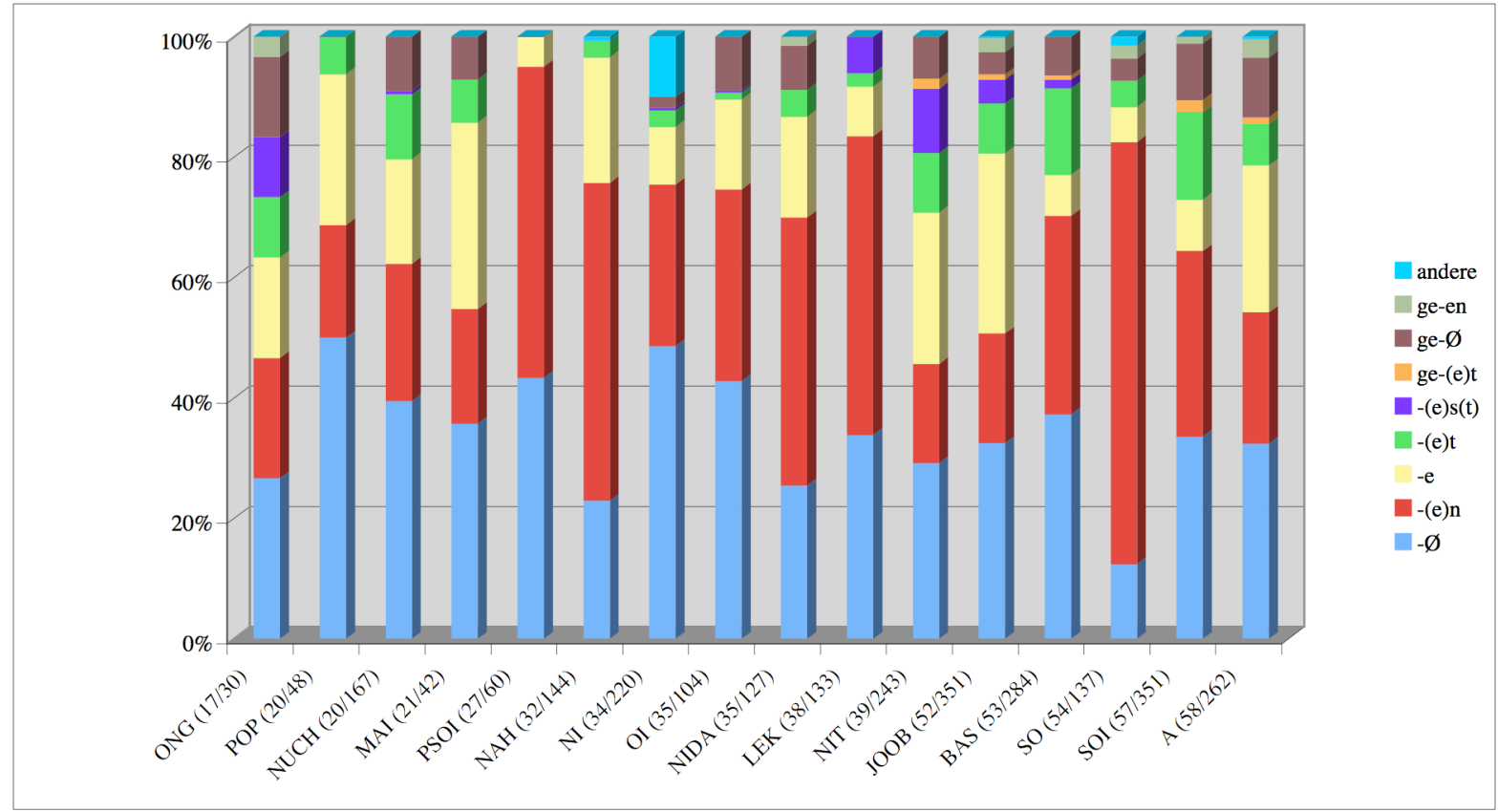

Die am häufigsten benutzten Flexionsmorpheme sind -en und $-\varnothing$. Sie gehen auf die Formen zurück, die im verbalen Flexionsparadigma am häufigsten vorkommen und für die meisten Kontexte anwendbar sind. Einige Abweichungen von den oben aufgelisteten üblichen verbalen Flexionsmorphemen im Deutschen lassen sich ebenfalls finden. Unter der Kategorie "andere", die nur einen minimalen Teil ausmacht, sind fehlerhafte Formen wie $-a, g e-+-e$ zusammengefasst. Das Flexionsmorphem ge- $+-\varnothing$ existiert im deutschen Verbparadigma nicht. Dieser Fall muss aber gesondert behandelt werden, weil es sich hier um eine Ausspracheabweichung handeln kann, die dadurch zu erklären ist, dass die Informantinnen überhaupt keine Konsonantencluster im Auslaut realisieren. Das Nullmorphem - $\varnothing$ könnte deshalb die Realisierung der intendierten Endung $-t$ sein.

Die Verwendung der 9 aufgelisteten Flexionsmorpheme schwankt bei den einzelnen Informantinnen zwischen 3 (PSOI) und 9 Varianten (JOOB). Im Rahmen dieser Studie wird viel Wert auf die Partizipien gelegt, weil die Erscheinung des Partizips Perfekt auf ein fortgeschrittenes Erwerbsniveau hinweist. ${ }^{7}$ Die Informantinnen können im Hinblick auf die Varianten ihrer Verbflexionsmorpheme in vier Gruppen eingeteilt werden:

- Die erste Gruppe mit minimaler Varianz der Flexionsmorpheme umfasst PSOI, POP, NAH und LEK. Bei ihnen treten 3 bis 5 verschiedene Endungen auf. Es gibt keine einzige deutliche Markierung des Partizips II (durch das Präfix ge-). PSOI hat zwar eine Verbform benutzt, die sich dem Partizip II zuordnen lässt (ich hab vergessen ... [PSOI-22]), aber bei ihr tritt nur diese einzige Verbform als Partizip II auf, die m. E. zu den Chunks zu zählen ist, während die anderen drei kein einziges Partizip bilden (vgl. Abbildung 1). Es handelt sich bei dieser Gruppe deshalb um Informantinnen mit ziemlich geringer Varianz der Flexion und ohne Partizip-Morphem.

\footnotetext{
7 Im Deutschunterricht wird die Bildung des Partizip II viel später eingeführt, deshalb tritt die Form im gesteuerten Sprachewerb erst in einer späteren Erwerbsphase auf (vgl. etwa Diehl et al. 2000: 364).
} 
in der Schweiz. Eine Bestandaufnahme.

- Mit 6 Personen (MAI, NI, SO, NIDA, OI und NUCH) bildet die zweite Gruppe die größte unter den Informantinnen. Bei dieser Gruppe mit 5-6 Varianten der Flexionsmorpheme lässt sich ein eindeutiges Zeichen für die Bildung des Partizips Perfekt finden, weil das Präfix ge- vorkommt, allerdings nur in Form von $g e-+-\emptyset$. Im Rahmen der vorliegenden Analyse wird dieses Morphem zwar als eine intendierte Form von $g e-+-t$ betrachtet und als ein anscheinend richtiges Flexionsmorphem gewertet, aber die Aussagekraft dieser Annahme bei dieser Gruppe ist eindeutig schwächer als bei den zwei folgenden Gruppen, da kein einziger Beleg für die richtige Form vorliegt.

- Zur dritten Gruppe gehören nur NIT und ONG. Beide benutzen 7 Varianten von Flexionsmorphemen. Sie unterscheiden sich allerdings von der zweiten Gruppe: Zum einen treten bei ihnen mehr Varianten auf, zum anderen gibt es bei ihnen Belege für richtige Formen der Partizipien. NIT gebraucht außer $g e-+-\emptyset$ ebenfalls das richtige Flexionsmorphem zur Partizipbildung ge- + $t$. Bei ONG lässt sich das Gegenteil finden. Ihre Verbformen im Partizip Perfekt werden zwar nicht mit $g e-+-t$, sondern mit ge- $+-\emptyset$ gebildet, aber bei ihr tritt das richtige Flexionsmorphem zur Partizipbildung bei starken Verben auf, das Morphem ge- + -en.

- Bei den restlichen 4 Informantinnen (SOI, A, BAS, und JOOB) lassen sich 7 bis 9 Varianten der Flexionsmorpheme feststellen. Diese Gruppe der Informantinnen zeichnet sich dadurch aus, dass hier nicht nur alle Varianten der Morpheme für finite Verbformen $^{8}$, sondern auch alle drei Varianten der Flexionsmorpheme für das Partizip Perfekt auftreten: $g e-+-\emptyset, g e-+-t$ und $g e-+-e n$. Die abweichende Form $g e-+-\varnothing$ lässt sich bei diesen Informantinnen plausibler dem intendierten Morphem $g e-+-t$ gleichsetzen - im Vergleich zu den anderen Informantinnen ohne diese richtige Form.

Im Hinblick auf die gebrauchten Flexionsmorpheme sowohl für finite als auch für infinite Verben sieht das Kontinuum des Erwerbsstands der Informantinnen folgendermaßen aus:

Abbildung 3: Erwerbsstand der Informantinnen im Hinblick auf Varianten der Flexionsmorpheme

\begin{tabular}{|c|c|c|c|}
\hline PSOI - POP - NAH - LEK - MAI - NI - SO - NIDA - OI - NUCH - NIT - ONG - SOI - A - BAS - JOOB \\
\hline 1. Gruppe & 2. Gruppe & 3. Gruppe & 4. Gruppe \\
\hline
\end{tabular}

Im Mittelpunkt dieses ersten Gesamtüberblicks über alle aufgetretenen Flexionsmorpheme steht die Größe der Varianz. Um festzustellen, ob die Verbflexion erworben wird, muss nun ein weiterer Aspekt in Betracht gezogen werden, nämlich ob diese Flexionsmorpheme richtig gebraucht werden.

\subsection{Richtig vs. falsch gebrauchte Flexionsmorpheme}

$\mathrm{Ob}$ es sich um richtig oder falsch gebildete Verbformen handelt, ist bei den unzuordenbaren Verbformen nicht feststellbar. Deshalb werden sie in dieser Phase ausgeschlossen. In den folgenden drei Abbildungen (Abbildung 4-6) wird die Verteilung aller vorkommenden

8 Bei dieser Annahme wird davon ausgegangen, dass die Endung - $t$ bei SOI und A wegen ihrer Ausspracheschwierigkeit eine Ersatzform für das Flexionsmorphem zur Markierung einer finiten Verbform in der 2. Pers. Sg. benutzt werden. D. h. sie reduzieren die Konsonantenanhäufung -st zunächst auf $-s$. Da diese beiden Informantinnen durchgehend kein Schluss-s artikulieren konnten, wird dieser reduzierte Auslaut weiter durch [ $\mathrm{t}\urcorner]$ ersetzt (ausführlicher zu Ausspracheabweichungen dieser Informantinnen vgl. Attaviriyanupap 2006). 
Flexionsmorpheme für drei Verbgruppen dargestellt: für finite Verben, Infinitive und Partizipien. Außer bei den Infinitiven, wo -en als das einzige richtige Flexionsmorphem gilt, wird immer jeweils der richtige (r) bzw. falsche (f) Gebrauch angegeben. Die Richtigkeit bezieht sich größtenteils auf die Affixe. Abweichungen in anderen Bereichen wie etwa falscher Vokal im Stamm sind äußerst selten und beeinträchtigen das Gesamtbild nicht. Deshalb werden solche Fälle zwar mitberücksichtigt, aber nicht gesondert dargestellt, sondern nur den falsch gebrauchten Formen zugeordnet. Die Daten werden jeweils im Hinblick auf die Anzahl der types bei jeder einzelnen Informantin und die Anzahl der tokens als 2. Sortierschlüssel von links nach rechts sortiert präsentiert.

Abbildung 4: Flexionsmorpheme bei finiten Verben

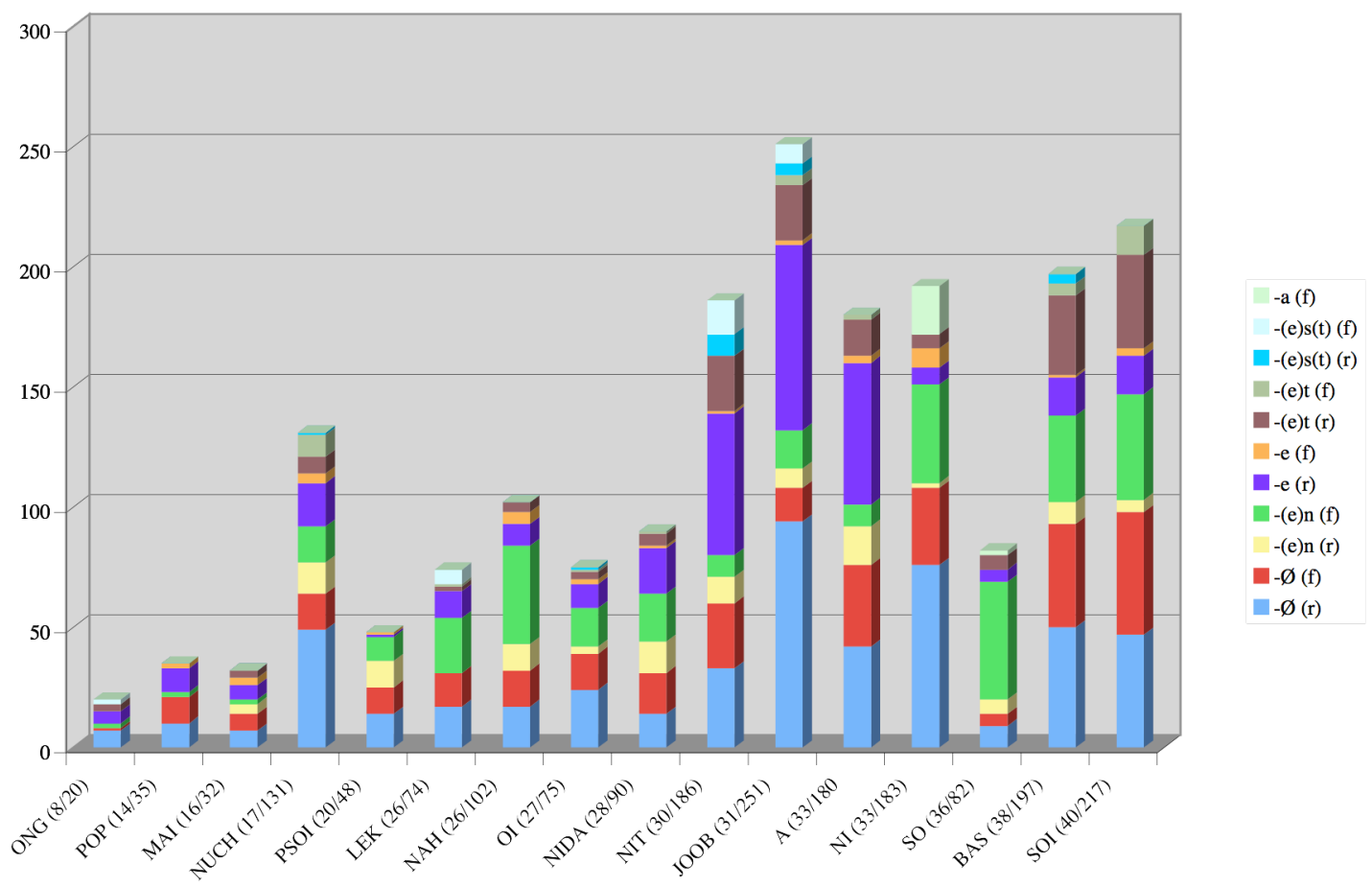


Abbildung 5: Flexionsmorpheme bei Infinitiven

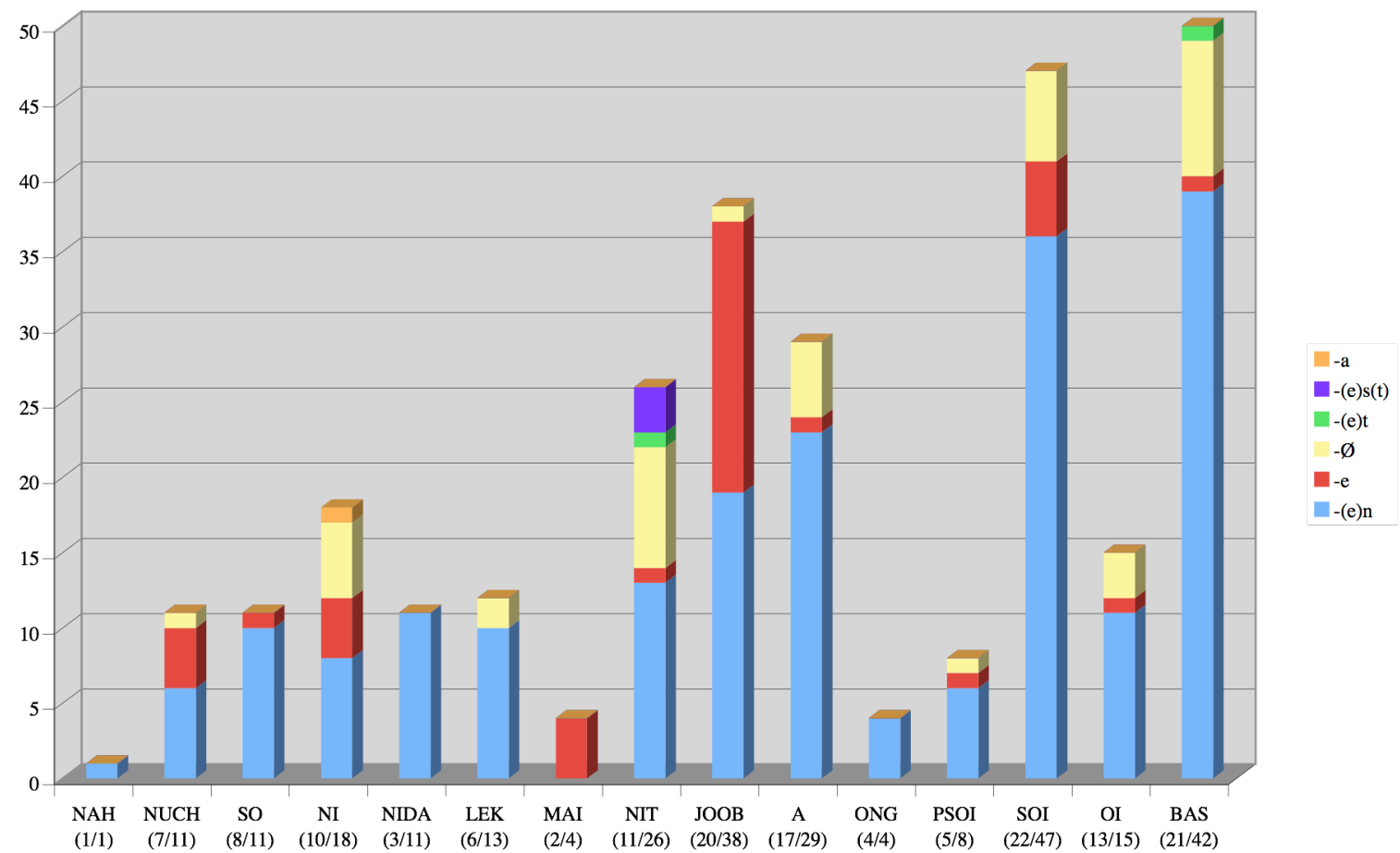

Abbildung 6: Flexionsmorpheme bei Partizipien

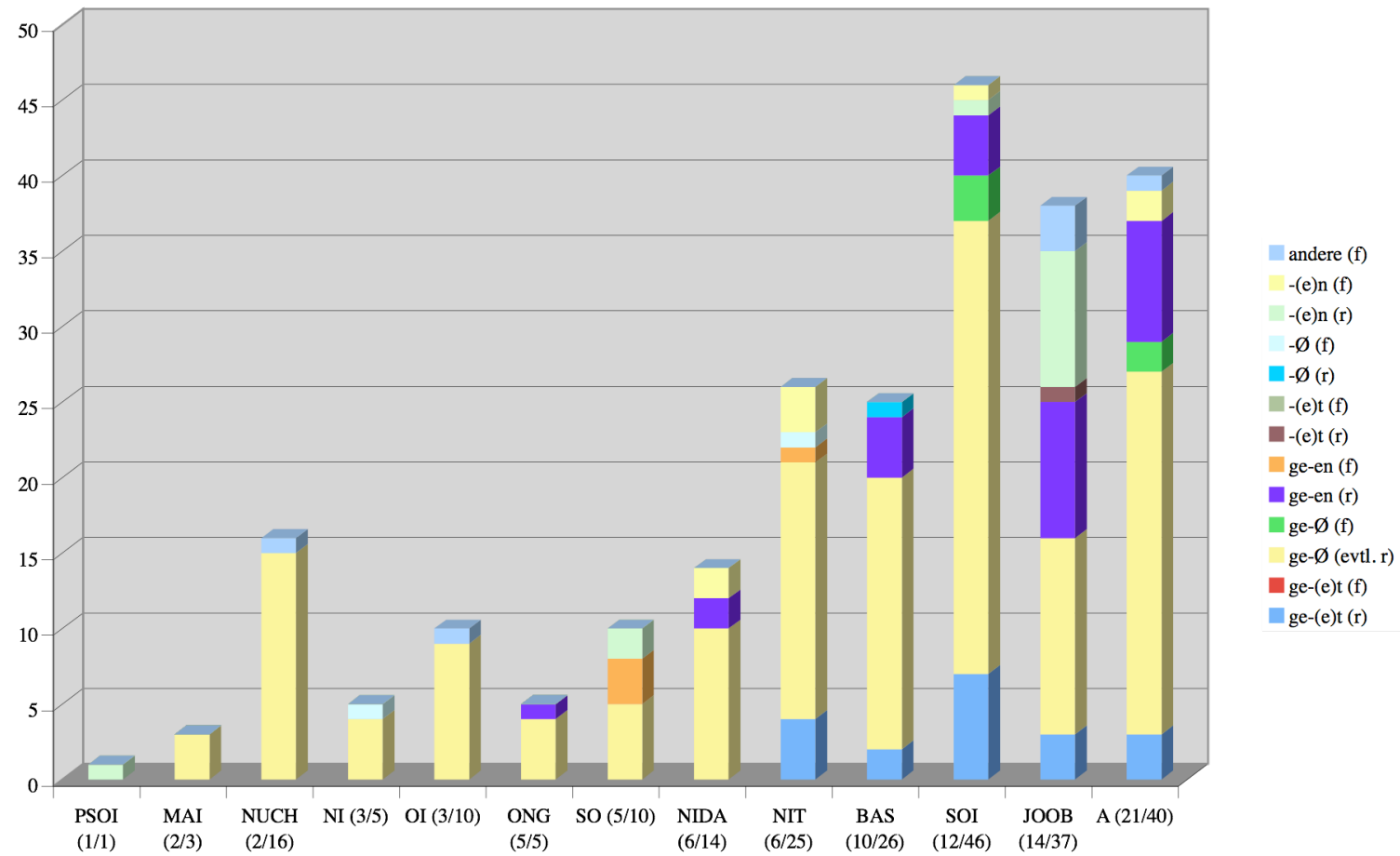

Die obigen Darstellungen vermitteln noch einmal das Bild, dass die Informantinnen die Flexionsmorpheme noch nicht systematisch gebrauchen. Insbesondere bei den intendierten finiten Verben scheint die Variation sehr groß zu sein. Dieselbe Tendenz besteht bei den Infinitiven, wo eigentlich nur -en als das einzige richtige Flexionsmorphem gilt. Auch hier treten verschiedene Endungen auf. Der Anteil von richtig gebrauchten Verbformen ist bei jeder Verbgruppe unterschiedlich. Um einen besseren Überblick zu ermöglichen, werden alle 
richtig verwendeten Flexionsmorpheme bei jeder Verbgruppe zusammengerechnet. Der prozentuale Anteil von richtig gebrauchten Flexionsmorphemen bei den finiten Verbformen im Vergleich zu den beiden Gruppen der infiniten Verbformen ist in der folgenden Tabelle zusammengestellt. Bei den infiniten Verbformen verwenden einige Informantinnen kein einziges Verb der jeweiligen Kategorie. Diese Spalten werden leer gelassen und ein wenig schattiert. Die Zahl 0.00 hingegen gibt einen prozentualen Anteil an, d. h. die Informantinnen haben mindestens eine infinite Verbform gebildet, aber keine einzige davon ist richtig.

Tabelle 2: Vergleich des prozentualen Anteils richtig gebrauchter Flexionsmorpheme bei intendierten finiten und infiniten Verbformen

\begin{tabular}{|l|r|r|r|}
\hline & finites Verb & Infinitiv & Partizip Perfekt \\
\hline SO & 31.71 & 90.91 & 80.00 \\
\hline NAH & 40.20 & 100.00 & \\
\hline LEK & 40.54 & 91.67 & 100.00 \\
\hline NI & 44.81 & 44.44 & 91.31 \\
\hline SOI & 49.31 & 76.60 & 100.00 \\
\hline PSOI & 54.17 & 75.00 & 90.00 \\
\hline OI & 54.67 & 73.33 & 85.71 \\
\hline NIDA & 56.67 & 100.00 & 100.00 \\
\hline BAS & 54.14 & 92.86 & 100.00 \\
\hline POP & 57.14 & & 93.75 \\
\hline MAI & 62.50 & 0.00 & 84.00 \\
\hline NUCH & 67.18 & 54.55 & 91.89 \\
\hline NIT & 72.58 & 50.00 & 100.00 \\
\hline A & 73.33 & 79.31 & 100.00 \\
\hline ONG & 75.00 & 100.00 & \\
\hline JOOB & 82.47 & 50.00 & \\
\hline
\end{tabular}

Die obige Tabelle zeigt Abweichungen des Anteils richtig gebrauchter Verbformen in jeder einzelnen Verbgruppe. Die Schwankung ist bei den Infinitiven am größten (von 0 bis 100\%). Allerdings bedarf auch dieses Ergebnis einer näheren Betrachtung. Erstens gilt die Endung - $e$ im Standarddeutschen zwar als eine falsche Endung für den Infinitiv, aber im Schweizerdeutschen ist sie richtig. Alle Informantinnen haben von ihrer realen sprachlichen Umgebung mehr Input mit dieser Endung bekommen, als in einer standardsprachlichen Umgebung zu erwarten wäre. Es ist deshalb nicht erstaunlich, dass diese Form als wichtigster Konkurrent zur korrekten Endung auftritt. MAI, die einzige Informantin, die gemäß der hochdeutschen Norm keine einzige richtige Verbform im Infinitiv bildet, gebraucht konsequent nur die Endung -e. Der Einfluss des Schweizerdeutschen kann deshalb nicht außer Acht gelassen werden, und der Wert 0.00 bedeutet nicht, dass alle Formen "völlig falsch" realisiert werden. Dieselbe Tendenz lässt sich auch bei 11 anderen Informantinnen beobachten. Zweitens gibt es ansonsten bei den Infinitiven im Vergleich zu den intendierten finiten Verben eindeutig weniger Abweichungen von der richtigen Endung. Wenn der Wert 0.00 aus dem genannten Grund herausgenommen wird, schwankt der Anteil der richtig gebrauchten Verbformen bei Infinitiven nur noch zwischen $44.44 \%$ und $100 \%$. Nicht zuletzt muss darauf hingewiesen werden, dass die Infinitive im Vergleich zu den intendierten finiten Verbformen zwar einen größeren Anteil der richtig gebrauchten Flexionsmorpheme aufweisen, aber die Zahl alleine ist noch kein eindeutiges Indiz für den Erwerb der Verbflexion, zumal -en im deutschen Sprachsystem nicht nur an sich polyfunktional ist, sondern auch bei allen Informantinnen eine zusätzliche Funktion als unmarkierte Form übernimmt.

Interessanter ist es, die Zahlen der anderen zwei Verbgruppen miteinander zu vergleichen. Der Anteil der richtigen Verbformen ist bei den Partizipien (80.00-100\%) deutlich höher als 
bei den intendierten finiten Verbformen (31.71-82.47\%). Es gibt allerdings drei Personen, die kein einziges Partizip Perfekt bilden. Diese Ergebnisse führen zu folgender Annahme: Sobald die Bildung eines Partizips Perfekt feststellbar ist, ist der Grad der Richtigkeit sehr hoch. Abbildung 7 soll dies verdeutlichen.

Abbildung 7: Prozentualer Anteil richtig gebrauchter Flexionsmorpheme bei finiten Verbformen im Vergleich zu dem bei den Partizipien

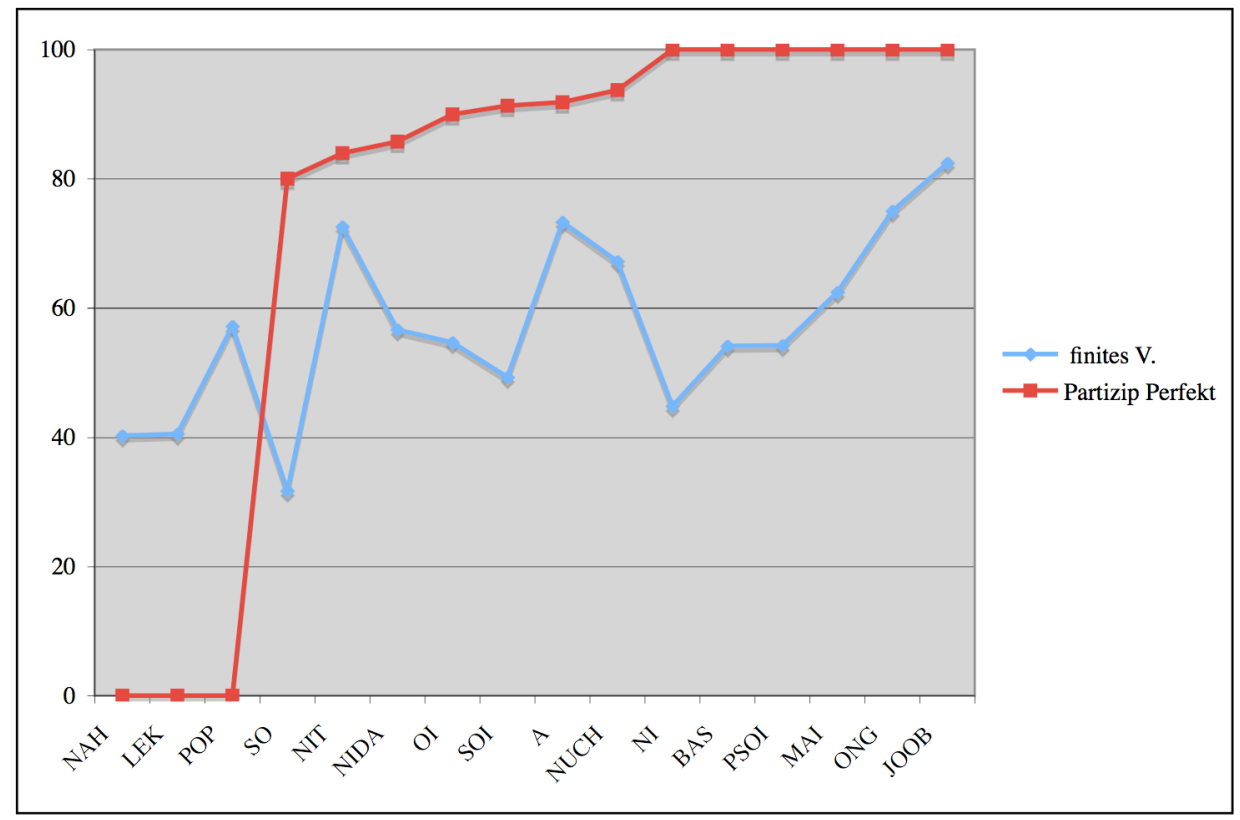

Trotz der Tatsache, dass es sich bei den ersten Erscheinungen von richtigen Partizipien - wie bei einem großen Anteil der richtig flektierten finiten Verbformen - ebenfalls um Chunks handeln kann, lässt sich aus diesen Daten eine deutliche Tendenz ablesen. Die Flexion infiniter Verbformen ist für die Informantinnen offensichtlich leichter zu beherrschen als die Personalflexion, die im Deutschen als das einzige morphologische Kriterium zur Unterscheidung finiter und infiniter Verbformen gilt. Im gesteuerten Unterricht wird in der Regel zunächst die Infinitivform als Nennform und der Gebrauch der Personalendung eingeführt. Erst viel später wird vermittelt, wie man das Partizip Perfekt bildet (vgl. auch Lehrwerke für Deutsch als Zweitsprache, z. B. Deutsch für die Schweiz ${ }^{9}$ ). Alle Informantinnen der vorliegenden Untersuchung erwerben die deutsche Standardsprache zwar nicht völlig natürlich, sondern in einem gemischten Kontext mit Unterricht, aber die Erwerbssequenz bei ihnen scheint überhaupt nicht von der Reihenfolge im gesteuerten Spracherwerb beeinflusst zu werden.

Um genauer festzustellen, welche Flexionsmorpheme einfacher, welche schwieriger zu erwerben sind, werden im Folgenden die korrekt gebrauchten Flexionsmorpheme hervorgehoben. Zunächst wird auf das Partizip Perfekt eingegangen, und im Weiteren auf die finiten Verbformen.

\footnotetext{
${ }^{9}$ Deutsch in der Schweiz, (Maurer 2001) gehört zu den Lehrwerken, die in der Schweiz im Deutschunterricht für ausländische Immigranten, auch für die Informantinnen der vorliegenden Untersuchung, häufig verwendet werden.
} 
Abbildung 8 : Verteilung der richtig gebrauchten Flexionsmorpheme bei Partizipien

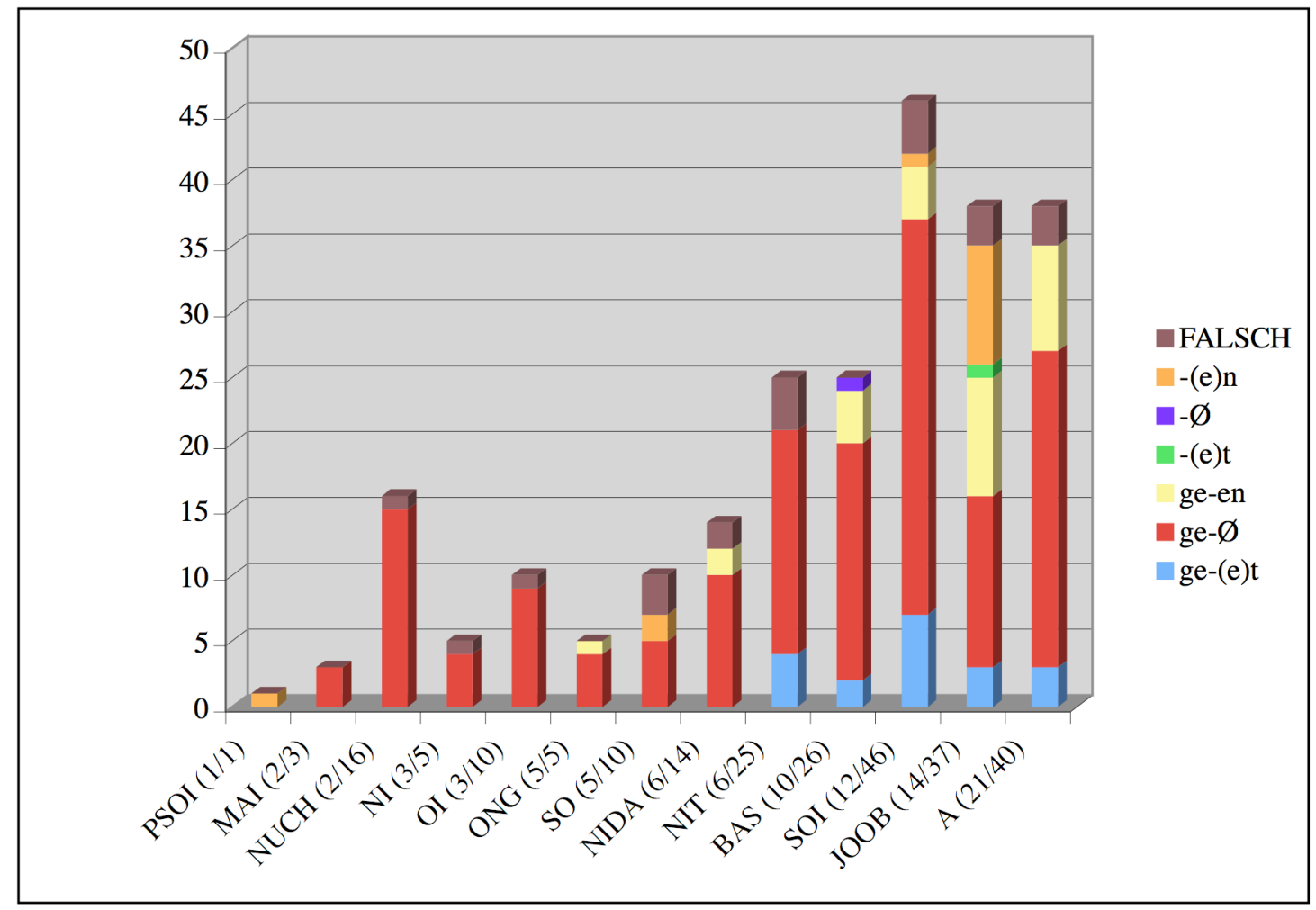

In dieser Hinsicht liegt bei den Partizipien ein interessantes Ergebnis vor. Außer bei PSOI ist bei allen anderen Informantinnen eine gemeinsame Tendenz feststellbar. Da PSOI nur ein einziges Partizip bildet und dieser Ausdruck von ihr (hab v vergessen) vermutlich zu den Chunks gehört, trägt die Abweichung bei ihr zu keiner Veränderung des Gesamtbildes bei. Der größte Anteil der oben als richtig betrachteten Verbformen betrifft ein Flexionsmorphem, das in der Zielsprache in der Tat gar nicht existiert $\left(g e^{-}+-\varnothing\right)$. Dieses wird nach Berücksichtigung der Ausspracheabweichung der Informantinnen aber als intendiertes richtiges Morphem betrachtet. Die damit intendierte Form ge- $+-t$ tritt hingegen nur bei 5 Personen auf und die Verbformen mit diesem richtigen Flexionsmorphem bilden nur einen kleinen Anteil an den gesamten Partizipien. Das Präfix $g e$ - als eine eigenständige Silbe begünstigt den Erwerb eines Teils des Flexionsmorphems zur Markierung der Form im Partizip Perfekt, so dass die $g e$-Form bzw. die eigenständige Silbe $g e$ - als einfach zu erwerben gilt. Dagegen erschwert das Schluss- $t$ die Aussprache, sobald der Laut einem anderen Konsonanten folgt und sich daraus ein Konsonantencluster ergibt. Beim Auftreten von fast allen Partizipien mit dem korrekten Flexionsmorphem $g e-+-t$ handelt es sich um einen Verbstamm ohne Koda, bei dem es hörbar ist, ob ein - $t$ artikuliert wird, auch wenn der Laut nicht plosiv gelöst wird, z. B. gehö $(r) t^{\urcorner}$(BAS, SOI, JOOB, A) oder geleh(r) $t^{\urcorner}$(A). In einem Konsonantencluster kommt das Suffix $-t$ des Partizips Perfekt - im Gegensatz zu $-t\urcorner-$ im ganzen Korpus nur ein einziges Mal bei BAS vor (kennengelernt).

Im Allgemeinen scheint dieses übliche Flexionsmorphem zur Bildung des Partizips Perfekt bei schwachen Verben am einfachsten und als Erstes erworben zu werden. Das Problem liegt bei der Aussprache. Konsonantencluster bleiben für diese Informantinnen ein tückisches Problem und verhindern zum großen Teil den Gebrauch der intendierten richtigen Verbformen. Das Flexionsmorphem ge- + -en für starke Verben ist m. E. nicht schwierig auszusprechen und sollte deshalb nicht schwieriger als das andere Morphem zu erwerben sein. Allerdings können wegen der Vokaländerung im Verbstamm falsche Formen gebildet werden. Trotzdem kann aus den vorliegenden Ergebnissen nicht eindeutig geschlossen werden, welches von den beiden Morphemen einfacher und früher erworben wird. Zum einen 
korreliert das Auftreten der Flexionsmorpheme in dem untersuchten Korpus mit der Anzahl der types, die jede einzelne Informantin produzierte. Zum anderen ist die Zahl der starken Verben im Deutschen, die im Partizip Perfekt mit dem Morphem ge- + -en gebildet werden müssen, viel geringer als die der schwachen Verben. Nur wenn es sich um einen rein gesteuerten Spracherwerb handelt, kann man sich auf die Reihenfolge bei der Vermittlung der Zielsprache stützen und plausibler den Schluss ziehen, dass die Partizipbildung bei schwachen Verben, d. h. mit ge- $+-t$ zuerst erworben wird. Das gleiche Kriterium gilt auch für die übrigen Verbformen, deren Partizipformen ohne das Präfix ge-, sondern nur entweder mit $-t$ oder -en gebildet werden. Nicht zuletzt sei angemerkt, dass sich im Korpus kein einziger Fehler finden lässt, bei dem ein Partizip eines starken Verbs mit dem für schwache Verben verwendeten Morphem $g e-+-t$ gebildet wird. Dagegen liegen einige Beispiele für die Bildung des Partizips Perfekt von schwachen Verben mit ge- + -en vor: gearbeiten (BAS), geheiraten und gekaufen (SO). Dies bestätigt noch einmal, dass der Unterricht, in dem zunächst immer die Flexion der schwachen Verben, das zweiteilige Morphem ge- + - $e) t$, vermittelt wird, so dass Lerner diese Regel häufig übergeneralisieren, auf den Erwerb der Partizipmorpheme keinen Einfluss ausübt.

Bei den finiten Verbformen ergibt sich ein ähnliches Bild, obwohl der prozentuale Anteil der richtig gebrauchten Flexionsmorpheme hier deutlich geringer ist. Anscheinend wenden die meisten Informantinnen keine Regeln an, die für die Mehrheit der Verben gelten, sondern gehen von gespeicherten Formen aus, weshalb starke Verben mehrheitlich mit einem richtigen Flexionsmorphem gebraucht werden. Außerdem scheint die Auswahl eines Flexionsmorphems noch ziemlich unsystematisch zu sein. Diese Tendenz lässt sich vor allem gut ablesen, wenn man die korrekt gebrauchten Morpheme bei finiten Verben den falsch ausgewählten gegenüberstellt, wie aus den beiden folgenden Abbildungen ersichtlich ist. Die Informantinnen werden in den beiden Darstellungen von links nach rechts nach Anzahl der types sortiert.

Abbildung 9: Verteilung der richtig gebrauchten Flexionsendungen bei finiten Verbformen

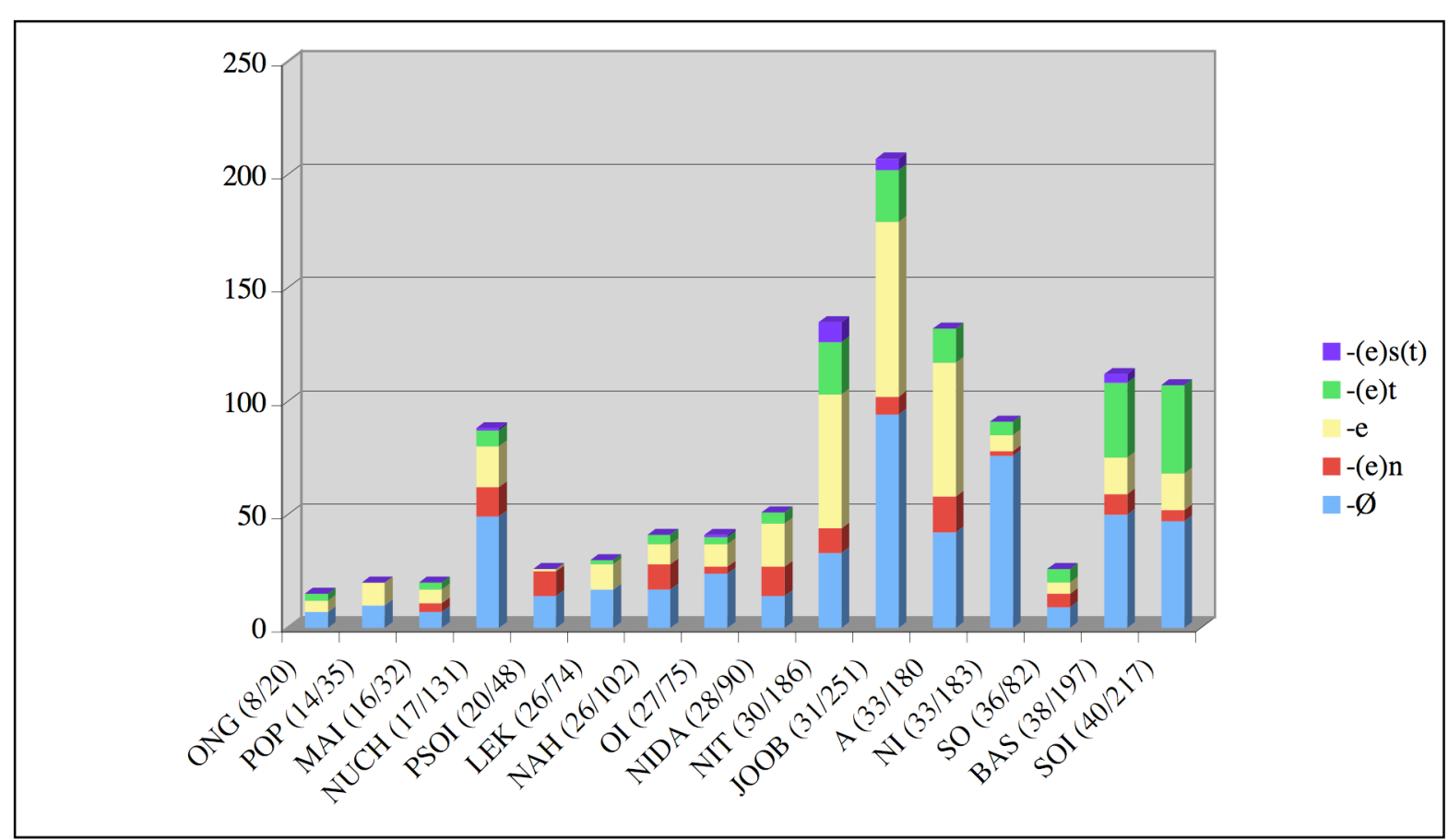


Abbildung 10: Verteilung der falsch gebrauchten Flexionsendungen bei finiten Verbformen

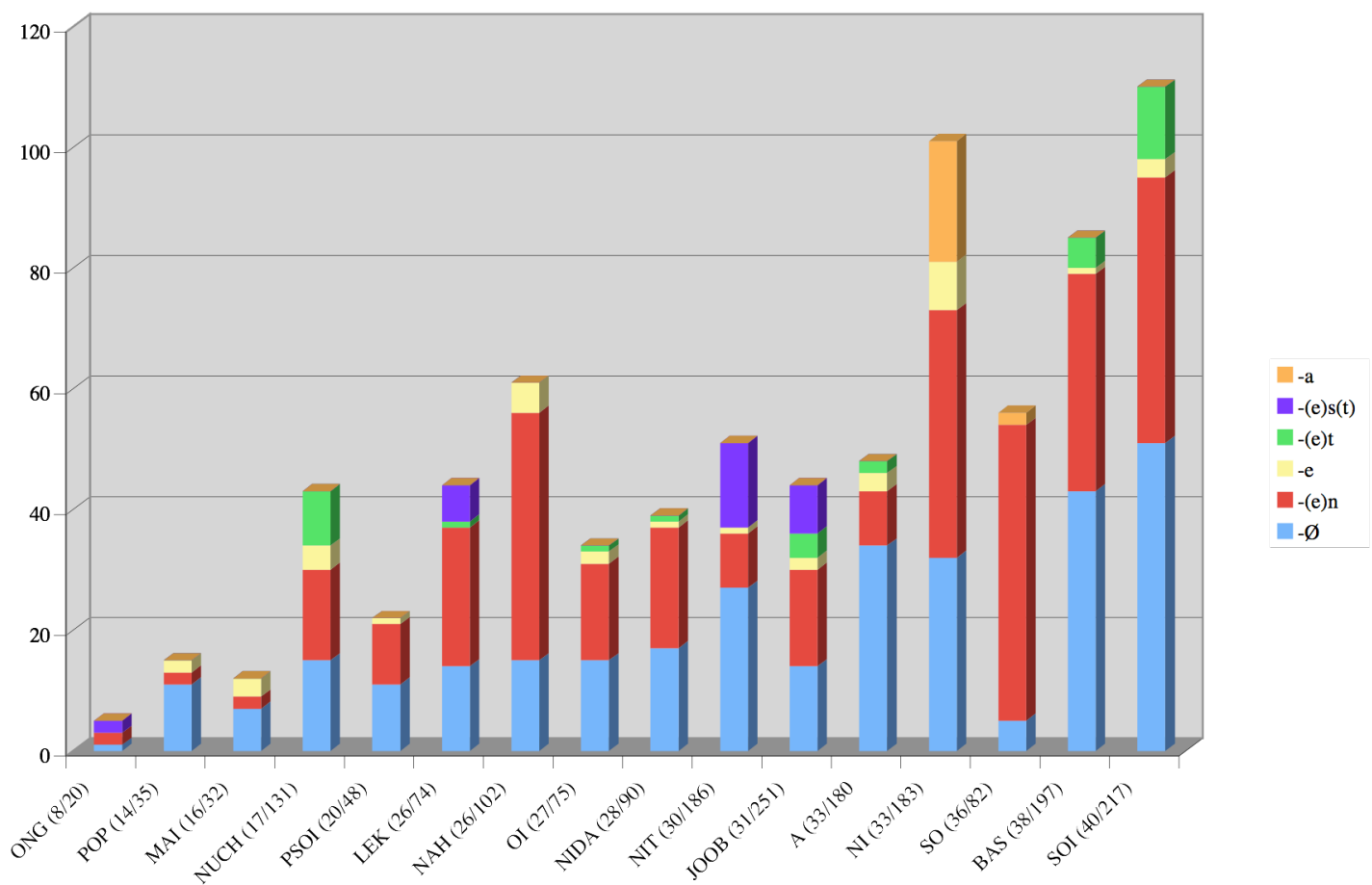

Das Nullmorphem wird bei den intendierten finiten Verben am meisten verwendet. Verben mit dieser Flexion bilden sowohl bei den richtigen als auch bei den falschen Verbformen die größte bzw. die zweitgrößte Gruppe. Beim falschen Gebrauch kann man von Übergeneralisierung sprechen, weil diese Endung ohnehin neben dem Morphem -en die am häufigsten gebrauchte Endung ist und als unmarkiert gilt. Bei den richtig gebildeten Verbformen handelt es sich allerdings nur um starke Verben, wo mehrheitlich ein Ablaut verlangt wird. Hier zeigt sich eine deutliche Tendenz: Verben mit Vokaländerung im Stamm werden wie neue Lexeme gespeichert. Weil sie im Alltag häufiger zu hören sind, werden sie mehrheitlich in der richtigen Form benutzt. Die Auswahl einer richtigen Endung setzt hingegen eine Systematik voraus. Deshalb werden die Endungen viel schwerer erworben, ungeachtet anderer Schwierigkeiten wie z. B. Ausspracheprobleme, vor allem was die Konsonantencluster betrifft.

Es hat sich herausgestellt, dass die Personalflexion im Vergleich zu den Partizipmarkierungen schwieriger zu erwerben ist. Im Folgenden wird deshalb der Erwerb der Personalflexion bei finiten Verben näher betrachtet.

\subsection{Erwerb der Personalflexion}

Alle finiten Verben im Deutschen müssen nach Person und Numerus konjugiert werden. Die beiden verbalen Kategorien werden bei finiten Verben nur durch ein Flexionsmorphem ausgedrückt und müssen immer mit dem Subjekt kongruieren. Im Deutschen unterscheidet man drei grammatische Personen (erste, zweite und dritte Person) und zwei Numeri (Singular und Plural). Die hier untersuchten Verbformen, die bei der Einordnung als finite Verben klassifiziert wurden, wurden deshalb gleichzeitig nach der Personalform des dazugehörenden Subjekts in sechs Gruppen eingeteilt. In Ergänzung zu der Frage, in welchem Ausmaß richtige Flexionsendungen verwendet werden, soll nun deshalb genauer betrachtet werden, ob die unterschiedliche Varianz der Personalendungen bei den einzelnen Informantinnen weitere Indizien liefert. Alle bei jeder einzelnen Personalform vorkommenden Personalendungen werden in Tabelle 3 aufgelistet. 
Tabelle 3: Personalflexion bei finiten Verbformen

\begin{tabular}{|c|c|c|c|c|c|c|c|c|c|c|c|c|c|}
\hline & $\begin{array}{c}1 . \\
\text { Pers. } \\
\text { Sg. }\end{array}$ & $\begin{array}{c}2 \\
\text { Pers. } \\
\text { Sg. }\end{array}$ & $\begin{array}{c}3 . \\
\text { Pers. } \\
\text { Sg. }\end{array}$ & $\begin{array}{c}1 . \\
\text { Pers. } \\
\text { Pl. }\end{array}$ & $\begin{array}{c}2 . \\
\text { Pers. } \\
\text { Pl. }\end{array}$ & $\begin{array}{c}3 . \\
\text { Pers. } \\
\text { Pl. }\end{array}$ & & $\begin{array}{c}1 . \\
\text { Pers. } \\
\text { Sg. }\end{array}$ & $\begin{array}{c}2 . \\
\text { Pers. } \\
\text { Sg. }\end{array}$ & $\begin{array}{c}3 . \\
\text { Pers. } \\
\text { Sg. }\end{array}$ & $\begin{array}{c}1 . \\
\text { Pers. } \\
\text { Pl. }\end{array}$ & $\begin{array}{c}2 . \\
\text { Pers. } \\
\text { Pl. }\end{array}$ & $\begin{array}{c}3 . \\
\text { Pers. } \\
\text { Pl. }\end{array}$ \\
\hline POP & $\begin{array}{l}-\mathrm{e} \\
-\varnothing \\
-\mathrm{en}\end{array}$ & $-\varnothing$ & -Ø & & & $-\varnothing$ & PSOI & $\begin{array}{l}-\mathrm{e} \\
-\varnothing \\
-\mathrm{en} \\
\end{array}$ & -Ø & -Ø & & & -en \\
\hline MAI & $\begin{array}{l}-\mathrm{e} \\
-\varnothing\end{array}$ & & $\begin{array}{l}-t \\
-\varnothing \\
-e \\
-e n\end{array}$ & -en & & $\begin{array}{l}\text {-en } \\
-e \\
-\varnothing\end{array}$ & SO & $\begin{array}{l}-e \\
-\varnothing \\
- \text { te } \\
\text {-en }\end{array}$ & $\begin{array}{l}-\varnothing \\
\text {-en } \\
\text {-a }\end{array}$ & $\begin{array}{l}-t \\
-\emptyset \\
-e n\end{array}$ & -en & & -en \\
\hline NAH & $\begin{array}{l}-\mathrm{e} \\
-\varnothing \\
-\mathrm{en}\end{array}$ & $\begin{array}{l}-\varnothing \\
\text {-en }\end{array}$ & $\begin{array}{l}\text {-et } \\
\text { - } \varnothing \\
\text {-en } \\
\text {-e }\end{array}$ & $\begin{array}{l}\text {-en } \\
-\mathrm{e}\end{array}$ & & -en & NI & $\begin{array}{l}-e \\
-\varnothing \\
-e n\end{array}$ & $\begin{array}{l}-\varnothing \\
\text {-en } \\
-\mathrm{e} \\
-\mathrm{a}\end{array}$ & $\begin{array}{l}-\mathrm{t} \\
-\varnothing \\
-\mathrm{en} \\
-\mathrm{e} \\
-\mathrm{a} \\
\end{array}$ & -en & & $\begin{array}{l}\text {-en } \\
-\varnothing \\
-e\end{array}$ \\
\hline LEK & $\begin{array}{l}-e \\
-\varnothing \\
-e n \\
-t\end{array}$ & & $\begin{array}{l}-t \\
-\varnothing \\
-e n \\
-s\end{array}$ & & & $-\varnothing$ & NIDA & $\begin{array}{l}-e \\
-\varnothing \\
\text {-en } \\
-t\end{array}$ & $-\varnothing$ & $\begin{array}{l}-t \\
-\varnothing \\
-e n \\
-e\end{array}$ & -en & & -en \\
\hline ONG & $\begin{array}{l}-\mathrm{e} \\
-\varnothing\end{array}$ & & $\begin{array}{l}-t \\
-\varnothing \\
-e n\end{array}$ & & & & SOI & $\begin{array}{l}\text {-e } \\
-\varnothing \\
\text {-en } \\
-t \\
\end{array}$ & $\begin{array}{l}-\varnothing \\
-t\end{array}$ & $\begin{array}{l}-t \\
-\varnothing \\
-e n \\
-e \\
\end{array}$ & -ent & & $\begin{array}{l}\text {-en } \\
-\varnothing \\
-t\end{array}$ \\
\hline $\mathbf{A}$ & $\begin{array}{l}-e \\
-\varnothing \\
-e n \\
-t \\
\end{array}$ & $\begin{array}{l}-\emptyset \\
-t\end{array}$ & $\begin{array}{l}-\mathrm{t} \\
-\varnothing \\
-\mathrm{e} \\
-\mathrm{en} \\
\end{array}$ & $\begin{array}{l}\text {-en } \\
\text {-Ø }\end{array}$ & & $\begin{array}{l}\text {-en } \\
-\varnothing \\
-e\end{array}$ & OI & $\begin{array}{l}\text {-e } \\
-\varnothing \\
\text {-en } \\
-t \\
\end{array}$ & $\begin{array}{l}-s \\
-\varnothing \\
-e n\end{array}$ & $\begin{array}{l}-t \\
-\varnothing \\
-e n \\
-e \\
\end{array}$ & & & -en \\
\hline BAS & $\begin{array}{l}-e \\
-\varnothing \\
-e n \\
-t\end{array}$ & $\begin{array}{l}-s \\
-\varnothing\end{array}$ & $\begin{array}{l}-t \\
-\varnothing \\
-e n\end{array}$ & & & $\begin{array}{l}\text {-en } \\
\text {-ten } \\
-\mathrm{e} \\
-\varnothing \\
-\mathrm{t}\end{array}$ & NUCH & $\begin{array}{l}-e \\
-\varnothing \\
-e n \\
-t\end{array}$ & $\begin{array}{l}-s \\
-\varnothing\end{array}$ & $\begin{array}{l}-t \\
-\varnothing \\
-e n\end{array}$ & $\begin{array}{l}\text {-en } \\
-e \\
-\varnothing\end{array}$ & & $\begin{array}{l}-e n \\
-e \\
-\varnothing \\
-t\end{array}$ \\
\hline NIT & $\begin{array}{l}-\mathrm{e} \\
-\varnothing \\
-e n\end{array}$ & $\begin{array}{l}-\mathrm{s} \\
- \text { sten }\end{array}$ & $\begin{array}{l}-t \\
-\varnothing \\
-e n \\
-s\end{array}$ & $\begin{array}{l}\text {-en } \\
\text {-ø }\end{array}$ & & $\begin{array}{l}\text {-en } \\
-\mathrm{e}\end{array}$ & JOOB & $\begin{array}{l}-e \\
-\varnothing \\
-e n\end{array}$ & $\begin{array}{l}-s t \\
-s \\
-\varnothing\end{array}$ & $\begin{array}{l}-t \\
-\varnothing \\
-e \\
-e n \\
-s\end{array}$ & $\begin{array}{l}\text {-en } \\
\text { - } \\
\text {-s }\end{array}$ & $-\mathrm{t}$ & $\begin{array}{l}\text {-en } \\
-\mathrm{e} \\
-\varnothing \\
-\mathrm{t} \\
-\mathrm{s}\end{array}$ \\
\hline
\end{tabular}

Die oben dargestellte Tabelle mag auf den ersten Blick den Eindruck erwecken, dass alle Informantinnen die Personalendungen völlig durcheinander bringen. Dies stimmt zum großen Teil auch. Nahezu in jeder Zelle, in der Verbformen vorkommen, sind mindestens zwei unterschiedliche Flexionsendungen vorhanden. Die anfänglich als "vermutlich" finit klassifizierten Verbformen weisen eher "scheinbare" Flexion bzw. Konjugation auf.

Aber auch die Anwesenheit verschiedener Varianten kann als Indiz für den Erwerb der Personalflexion angesehen werden. Im Hinblick auf die Varianz der Personalendung für intendierte finite Verbformen lassen sich die Informantinnen in drei Gruppen je nach Erwerbsstufe einteilen: 
1) Die erste Stufe betrifft zwei Personen. Hier tritt die geringste Varianz der Personalendungen auf. POP und PSOI bilden Verbformen mit nur wenigen Varianten bei den Flexionsendungen: - en, $-\varnothing$ und -e. Vorwiegend tritt nur der Stamm $(-\varnothing)$ auf. Die beschränkte Anzahl der Varianten von Flexionsendungen impliziert das niedrigste Niveau. Die Verben tragen nur eine lexikalische Bedeutung und keine grammatische Funktion. Dies führt häufig zu falschen Formenbildungen, weil diese drei Endungen noch keine grammatische Funktion haben. Dass nur wenige Varianten der Flexionsendungen vorkommen, kann ebenfalls als Indiz dafür gewertet werden, dass nicht wirklich ein Versuch vorliegt, die richtige Endung auszuwählen.

2) Zur nächsten Erwerbsstufe gehören diejenigen, bei denen noch eine zusätzliche Flexionsendung auftritt (MAI, SO, NAH, NI, LEK, NIDA, ONG). Es handelt sich um die Endung $-t$ zur Markierung der 3. Pers. Sg. Diese neue Endung könnte auch zu den Chunks gehören, weil sie nur für die 3. Pers. Sg. und nur für einige Verben gebraucht wird. ${ }^{10}$ Bei LEK und NIDA lässt sich - $t$ zwar ebenfalls bei einer anderen Personalform (1. Pers. Sg.) finden, aber es handelt sich bei den beiden Fällen nur um den wiederholten Gebrauch der einen Konstruktion ich hat. Ansonsten gibt es keinen weiteren Beleg, so dass die beiden trotzdem der zweiten Gruppe zugeordnet werden müssen. LEK gebraucht bei der 3. Pers. Sg. außerdem noch eine weitere Endung. Die Endung $-s$ bei ihr zeigt aber deutlich eine falsche Übergeneralisierung des Schluss-s, was besonders ihr individuelles Ausspracheproblem ist. Dadurch, dass sie die 2. Pers. Sg. überhaupt nicht als Subjekt gebraucht, weist die Endung $-s$ eindeutig auf einen Aussprachefehler hin und die Informantin zählt deshalb noch zur zweiten Gruppe. Interessanterweise kommt bei NI und SO eine spezielle Endung vor: Die Endung - $a$ ist überhaupt nicht im Flexionsmorpheminventar des Deutschen vorhanden, kann aber eine Ausspracheabweichung von $-e$ sein.

3) Die dritte Gruppe weist eine weitere Flexionsendung auf, die im Verbparadigma des Deutschen die Funktion hat, die zweite Person Singular zu markieren. Sie kommt allerdings in zwei Varianten vor: $-s t$ und $-s$. Das richtige Flexionsmorphem -st lässt sich nur bei JOOB finden, ansonsten wird durchgehend die etwas abweichende Variante $-s$ verwendet. Da die thailändischen Informantinnen Konsonantencluster im Auslaut meistens nicht realisieren (vgl. mehr dazu in Attaviriyanupap 2006), ist es nicht erstaunlich, dass -st kaum zu hören ist. Darüber hinaus unterscheidet sich diese Gruppe der Informantinnen durch die Verwendung der Endung $-s$ deutlich von den anderen bis dahin besprochenen Gruppen. Die Informantinnen SOI, A, OI, BAS, NUCH, NIT und JOOB gehören alle zu dieser letzten Gruppe, weil kein weiteres Merkmal besteht, mit dem einige von ihnen im Hinblick auf die Verbendung in eine höhere Erwerbsstufe eingeordnet werden könnten. Genauer betrachtet können diese 7 Informantinnen zwar noch weiter in drei Subgruppen eingeteilt werden, aber trotz dieser Einteilung kann nicht festgestellt werden, welche Gruppe einem niedrigeren welche einem höheren Erwerbsniveau zuzuordnen ist.

OI, BAS und NUCH benutzen die Endung $-s$ nur für die 2. Pers. Sg. und daneben noch die unmarkierte Form - $\varnothing$ (sowie, nur OI, -en). Da die Verbformen in der 2. Pers. Sg. eher selten als Chunks zu charakterisieren sind (wie es bei der 1. und 3. Pers. Sg. öfter der Fall ist), weist das Auftreten dieser Endung bei nur einer Personalform nicht unbedingt auf eine niedrigere Erwerbsstufe hin als Fälle, in denen sie auch bei anderen Personalformen auftreten.

JOOB und NIT benutzen beide die Endung $-s$ (JOOB auch $-s t$ ). Der Gebrauch beschränkt sich aber nicht nur auf die 2. Pers. Sg. JOOB produziert $-s$ auch bei der 3. Pers. Sg. und 3. Pers.

\footnotetext{
10 Das Fehlen abweichenden Gebrauchs dieser Flexionsendung bei anderen Personen und Numeri dürfte implizieren, dass die Informantinnen gehörte Verbformen aus dem Gedächtnis reproduzieren, ohne die Verblexion zu bearbeiten (vgl. die Beschreibung des Chunk-Phänomens z. B. bei Diehl et al 2000: 136).
} 
Pl., während NIT $-s$ auch für die 3. Pers. Sg. einsetzt. Die beiden Informantinnen gebrauchen parallel zu dieser Endung auch das generalisierte Morphem - $\emptyset$. Bei NIT kommt die Endung -en (bzw. -sten) bei einem Lexem vor: möchsten (2 Mal). Es scheint ihr also bewusst zu sein, dass bei der 2. Pers. Sg. die Lautfolge -st hinzugefügt werden muss. Das Wissen über die Verwendung der Endung -st für die 2. Pers. Sg. ist deshalb bei NIT und JOOB deutlich erkennbar, vor allem bei JOOB, weil sie die einzige Person ist, die den Konsonantencluster -st im Auslaut bei einer Verbform für die 2. Pers. Sg. richtig realisiert (hast, bist, musst). Trotzdem ist anzumerken, dass die Endung $-s$ bei JOOB und NIT auch als Übergeneralisierung in der Aussprache betrachtet werden kann, weil die beiden, im Unterschied zu den meisten Informantinnen, das Schluss-s mehrheitlich realisieren.

Dagegen tritt, wie die obige Tabelle zeigt, die richtige Endung für die 2. Pers. Sg. weder bei SOI noch bei A auf. Allerdings gibt es hier einen triftigen Grund, die beiden dennoch der Gruppe mit dieser Endung zuzuordnen. SOI und A setzen zwar nirgendwo die Endung $-s(t)$ ein, aber bei ihnen tritt stattdessen die Endung - $t$ bei der 2. Pers. Sg. auf, was bei den anderen Informantinnen, die diese Endung realisieren, nicht zu beobachten ist. Dies liegt m. E. daran, dass diese beiden Informantinnen im ganzen Korpus $-s$ im Auslaut kaum realisierten. Die Erscheinung der Endung $-t$ bei der 2. Pers. Sg. könnte deshalb die Endung $-s$ bzw. $-s t$ implizieren, die zunächst - wie bei den anderen Informantinnen dieser Gruppe - auf $-s$ reduziert wird und durch ihre Unfähigkeit $-s$ im Auslaut zu sprechen zum - $t$, genauer zum [ $t\urcorner]$, wird. Diese Markierung der 2. Pers. Sg. ist bei ihnen außerdem durch die Abwesenheit der sonst unmarkierten Endung -en zu begründen. Bei den meisten Informantinnen der Gruppe mit der Personalendung - $(e) s(t)$ verschwindet die sonst übergeneralisierte Endung -en bei der 2. Pers. Sg. Dieses Phänomen hebt vor allem die Bedeutung des Auftretens der Personalendung - $(e) s t$ im Erwerb der Personalflexion hervor.

Die hier aufgestellten Erwerbsstufen stimmen mit den Resultaten von Köpcke (1987) überein, der den ungesteuerten Erwerb morphologischer Ausdrucksmittel durch L2-Lerner am Beispiel der Personalflexion untersuchte. Er hat vier Erwerbsphasen festgestellt: 1. Phase: Vorkommen von nur $-\varnothing$, $-e$ und $-(e) n, 2$. Phase: $-t$ markiert die 3. Pers. Sg.; 3. Phase: $-s t$ markiert die 2. Pers. Sg.; 4. Phase: keine Übergeneralisierung von $-\varnothing,-e$ und $-(e) n$ :

Ein Lerner, zu dessen Repertoire die Form -st zählt, wird auch die Form - $t$ produzieren, und ein Lerner, der die Form - $t$ produziert, weist in seinem Formenrepertoire auch die Formen $-e,-\varnothing$ und -(e)n auf. Diese Abfolge gilt jedoch nicht umgekehrt. (Köpcke 1987: 194f.)

Das Resultat der vorliegenden Analyse zeigt genau diese Abfolge, wobei sich die fortgeschrittenste Gruppe erst in der 3. Phase befindet. Um das Kongruenzsystem vollständig $\mathrm{zu}$ erwerben, müssen Lernende die 4. Phase erreichen, was keine der thailändischen Informantinnen bislang geschafft hat.

Dass das Flexiv der 3. Pers. Sg. (-(e)t) als die erste Variante neben den unmarkierten Endungen auftritt, stimmt mit Ergebnissen vieler Studien zum Erst- und Zweitspracherwerb überein (vgl. auch den Überblick darüber in Diehl et al. 2000 127ff.). Allerdings tendiert auch diese Endung, wie die anderen erwähnten übergeneralisierten Flexive (-en, - $\emptyset$ und $-e)$ dazu, die Funktion einer unmarkierten Form zu übernehmen. Und nicht zuletzt kann dieses Flexiv bei den thailändischen Informantinnen ebenfalls als Abweichung von $-s$ bzw. als zunächst reduzierte und dann noch abweichende Form der Konsonantenverbildung -st im Anlaut betrachtet werden.

Das Flexionsmorphem -st gilt als die markierteste Endung im ganzen Flexionsparadigma der Verben. Während die meisten Flexionsendungen polyfunktional sind, ist die Endung -st allein für die 2. Pers. Sg. reserviert. Wenn diese Endung einmal auftritt, dann ist zu erwarten, dass sie kaum für andere Personalformen übergeneralisiert wird. Ihre Erscheinung hängt außerdem 
mit dem Abbau der übergeneralisierten Flexionsmorphemen, vor allem -en, wie aus Tabelle 3 ersichtlich ist, zusammen. Das Auftreten dieser Endung ist deshalb ein Zeichen für ein fortgeschrittenes Erwerbsniveau, weil sie immer erst später auftritt. In dieser Hinsicht schließe ich mich Köpcke (1987: 202) an, der argumentiert, dass erst mit dem Erwerb der Form -st einschließlich deren Bedeutung im Kongruenzsystem der Weg für die morphologische Markierung von Person und Numerus geebnet wird. Die Informantinnen in der letzten Gruppe befinden sich in einer Phase, in der dieses entscheidende Flexiv auftritt, allerdings noch nicht systematisch genug angewendet wird. Anscheinend haben sie die Erwerbsphase der Subjekt-Verb-Kongruenz noch nicht abgeschlossen oder es handelt sich bei den Fortgeschrittenen um Fossilierung.

Da die untersuchten Verbformen größtenteils noch in invarianten Formen auftauchen, gibt es für die meisten Verben noch keinen paradigmatischen Kontrast, was die Analyse einigermaßen erschwert. Es ist schwierig herauszufinden, ob die Informantinnen systematische Oppositionen zwischen den $\mathrm{zu}$ markierenden grammatischen Personen aufstellen. Aus diesem Grund wurde bei der Analyse mehr Wert auf die Varianten der Flexive im Allgemeinen und im Hinblick auf dieselben Lexeme gelegt. Dass sich dabei ähnliche Resultate wie in anderen Untersuchungen ergeben haben, ist allerdings ein deutliches Indiz dafür, dass der Hochdeutscherwerb der thailändischen Immigrantinnen ebenfalls systematisch verläuft. Die Erwerbssequenz im natürlichen Kontext (vgl. die Übereinstimmung mit Köpckes Ergebnissen) scheint eine größere Rolle zu spielen als der Unterricht und als der Einfluss der Muttersprache, denn im Unterricht wie auch in der Muttersprache ist keine bestimmte Reihenfolge der zu erwerbenden Personalendungen vorgesehen.

Es soll aber an dieser Stelle erneut hervorgehoben werden, dass es im vorliegenden Fall problematisch ist, die Äußerungen im Hinblick auf den Erwerb der Flexionsendung -(e)st zu analysieren, vor allem aufgrund der Ausspracheprobleme der Informantinnen. Während einige Informantinnen das Schluss-s übergeneralisieren (vor allem LEK und NIT), lässt sich ebenfalls eine gegenläufige Tendenz feststellen, nämlich dass einige Personen diesen Auslaut fast nie realisieren (vor allem SOI und A). Besonders schwierig zu analysieren ist dies bei Verben, deren Stamm auf $-s$ endet. Hier ist nicht feststellbar, ob die jeweilige intendierte Verbform nur den Stamm enthält oder ebenfalls eine Flexionsendung und im letzteren Fall welche Endung, vgl. etwa mut ${ }^{\urcorner}$bzw. muss und wei( $(\beta)$, die im Korpus bei der 1., 2. wie auch 3. Pers. auftreten können. Ebenso verhält es sich bei der Endung der Verbformen bei der 2. Pers. Sg., die als [ $[\mathrm{t}]$ realisiert wurden. Es ist keineswegs klar, was tatsächlich als Basis dieser Realisierung diente. Diesem Auslaut kann, vor allem wenn man den Einfluss der diglossischen Situation mitberücksichtigt, eine von drei verschiedenen intendierte Formen zugrunde liegen: [st], [t] oder [j]. Die letzte Variante geht auf Schweizerdeutsch zurück, wo die Endung für die 2. Pers. Sg. mit einem Frikativ realisiert wird (z. B. du geisch im Berndeutschen). Aufgrund der Hauptschwierigkeiten der thailändischen Immigrantinnen in Bezug auf Auslautkonsonanten und Konsonantencluster können alle diese drei Endungen zusammengefasst und als ungelöstes $-t$ realisiert werden.

\subsection{Flexionsanalyse der 10 am häufigsten gebrauchten Verben}

Der als letzter zu behandelnde Aspekt ist der Vergleich der Flexion im Hinblick auf den Unterschied zwischen verschiedenen Verbtypen. Von allen 153 Verben (types), die im Korpus vorkommen, vertreten die 10 am häufigsten aufgetretenen Verben zufällig im Hinblick auf ihr Flexionsparadigma unterschiedliche Verbtypen, deshalb werden sie im Folgenden gesondert behandelt. Die Häufigkeit bezieht sich hier auf die Anzahl der 
in der Schweiz. Eine Bestandaufnahme.

Informantinnen, die diese Verben gebrauchen. ${ }^{11}$ Diese Anzahl wird in Klammern nach jedem einzelnen Verb angegeben. Die betroffenen Verben lassen sich in vier Gruppen einteilen:12

1) arbeiten (13), machen (16), sagen (14): Die erste Gruppe besteht aus regelmäßigen Verben. Sie werden im Präsens regelmäßig konjugiert (mit $-e,-(e) s t,-(e) t$, -en, - $t$ und $-e n$ ) und enthalten bei der Konjugation keine Vokaländerung im Stamm. Das Partizip Perfekt bei diesen drei Verben wird mit $g e-+-(e) t$ gebildet.

2) gehen (13), kommen (13), sprechen (16): Die zweite Gruppe besteht aus unregelmäßigen Verben. Diese Verben bilden das Paradigma (Präsens - Präteritum - Perfekt) mit Ablaut. Das Partizip Perfekt wird durch Hinzufügen des Flexionsmorphems ge- + -en gebildet. Allerdings besteht ein Unterschied im Flexionsparadigma dieser Verben im Präsens. Es gibt im Präsens keine Vokaländerung bei gehen und kommen, sondern nur bei sprechen (er spricht).

3) können (15), wissen (15): Diese beiden Verben werden von Helbig/Buscha (2001: 28f.) einer besonderen Gruppe der regelmäßigen Verben zugeordnet. Trotz der Vokaländerung wird ihr Partizip Perfekt mit $g e-+-t$ gebildet. ${ }^{13}$ Deshalb werden sie der dritten Gruppe zugeordnet.

4) haben (15), sein (16): Die letzte Verbgruppe umfasst die beiden Verben, die im Deutschen auch als Hilfsverb fungieren. Sie weisen zwar unterschiedliche Flexionstypen auf, haben aber ihre bedeutende Rolle als Hilfsverben im Deutschen gemeinsam. Der Gebrauch dieser beiden Verben zeigt naturgemäß die höchste Frequenz (vgl. auch Ruoff 1981: 481). Deshalb sollen sie getrennt von anderen Gruppen behandelt werden, denn es ist anzunehmen, dass mit diesen Verben am meisten richtige Verbformen gebildet werden.

Ausgehend von den bisher besprochenen Ergebnissen der Korpusanalyse kann eine Hypothese aufgestellt werden: Der Grad des Erwerbs der Verbalflexion ist bei der 4. Gruppe am höchsten und bei der 1 . Gruppe am niedrigsten. In der folgenden Analyse werden unzuordenbare Verbformen ausgeschlossen.

\section{Gruppe}

- arbeiten: Das Verb kommt zwar sehr häufig vor, aber mehrheitlich in falschen Formen. Bei den fehlerhaften Formen im Partizip Perfekt fehlt entweder das ge- oder es wird mit dem Flexionsmorphem eines starken Verbs kombiniert (gearbeiten). Nur als Infinitiv kommt das Verb arbeiten mehrheitlich mit der richtigen Endung -en vor, allerdings nur bei 3 Personen ohne Abweichungen. Erstaunlicherweise wird das Verb nur ein Mal mit der richtigen Endung der 3. Pers. Sing. gebildet (arbeitet $\urcorner$ bei NIT), obwohl das Verb arbeiten im Korpus häufig in der ersten und dritten Pers. Sg. verwendet wird. Auch für die 1. Pers. Sing. gibt es nur jeweils eine korrekte Verbform bei NUCH und A (arbeite). Ansonsten wird -en als eine generalisierte Endung für dieses Verb eingesetzt. Auffällig ist weiterhin, dass das Nullmorphem als das zweithäufigste Flexiv auftritt, obwohl die Flexion bei diesem Verb eigentlich immer eine zusätzliche Silbe bedingt und keine Ausspracheschwierigkeiten bereiten sollte. Der Status von arbeiten als Verb bei den Informantinnen ist deshalb in der Tat noch fraglich, weil arbeiten

\footnotetext{
11 Wenn man diese Verben in einem Frequenzwörterbuch nachschlägt, lassen sie sich alle auf jeden Fall unter den Grundverben mit sehr hoher Häufigkeit finden (vgl. z. B. Ruoff 1981).

12 Die folgende Einteilung beruht auf gängigen Ansätzen, die man im Unterricht Deutsch als Fremdsprache verwendet (cf. z. B. Helbig/Buscha 2001: 30ff.).

13 Modalverben und wissen weisen mehrere Besonderheiten auf, die mit der Tatsache im Zusammenhang stehen, dass ihr heutiges Präsens ursprünglich ein Präteritum war. Es handelt sich um sog. Präterito-Präsentia (cf. Helbig/Buscha 2001: 29; Hentschel/Weydt 2003: 73).
} 
kein transitives Verb ist und deshalb nicht eindeutig vom Substantiv Arbeit zu unterscheiden ist, solange nur der Stamm als Verbform auftritt. ${ }^{14}$

- machen: Das Verb machen kommt bei allen Informantinnen vor. Bei diesem Verb treten auch Partizipien auf, die mehrheitlich als gemach und nur ein einziges Mal von JOOB richtig als gemacht realisiert werden. Die Form gemach ist durch das stark markierte Präfix ge- als richtig zu betrachten. Problematisch ist die Form mach, die häufig vorkommt. Während diese endungslose Verbform als falsch gilt, ist es allerdings möglich, dass sie bei der 3. Pers. Sg. anstatt von macht gesprochen wird, weil die Informantinnen Konsonantencluster im Auslaut nicht realisierten. Dies bleibt aber ungeklärt, weil sich der Gebrauch dieser Verbform nicht auf die dritte Person beschränkt. Allerdings ist das ein Hinweis auf ein wichtiges Problem: die Aussprache verhindert den Ausdruck einiger Flexionsmorpheme.

- sagen: Für dieses Verb lassen sich Partizipien finden, die ausschließlich mit $g e-+-\varnothing$ gebildet werden. Es handelt sich um dasselbe Phänomen wie bei machen. Unter den finiten Verbformen lassen sich weder die Endung $-s$ noch $-t$ finden. Wegen eines größeren Anteils an Partizipien erhöht sich der gesamte Anteil der richtig gebrauchten Flexionsmorpheme enorm, obwohl eigentlich bei den finiten Verbformen fast die gleiche Tendenz vorliegt wie bei den anderen zwei schwachen Verben. Es gibt vor allem bei den intendierten finiten Verben noch viele abweichende Varianten. Und wenn man sag bei der 3. Pers. Sg. als eine richtig intendierte, aber falsch ausgesprochene Form betrachtet, dann kann der Anteil der richtig gebrauchten Flexionsmorpheme noch ein wenig nach oben korrigiert werden.

\section{Gruppe}

- gehen: Hier liegt kein einziges Partizip Perfekt vor. Allerdings kann man vermuten, dass JOOB das Partizip von gehen bilden kann, weil bei ihr die Verbform weggegangen einmal vorkommt. Die Infinitive des Verbs kommen bis auf eine einzige Ausnahme alle mit der richtigen Endung vor. Obwohl die richtigen infiniten Formen keinen großen Anteil an der Gesamtzahl der Formen dieses Verbs ausmachen, kommen bei gehen mehr richtige Verbformen vor als bei den regelmäßigen Verben in der ersten Gruppe. Die Endung - $e$ kommt nur bei der 1. Pers. Sg. vor, während -en bei allen Kategorien gebraucht wird. Auch der Stamm ohne Endung lässt sich finden, allerdings viel seltener als bei anderen Verben. Dies korreliert mit der Silbenstruktur des Verbstamms. Da geh-keine Koda enthält, lässt sich die Endung - $t$ leichter feststellen und deutlich von $-\varnothing$ unterscheiden. Die Endung $-t$ tritt allerdings nicht nur bei der 3. Pers. Sing. auf, sondern wird z. T. übergeneralisiert. Nur bei der 1. Pers. Sg. lässt sich diese Endung nicht finden. D. h. bei diesem Verb wird zunächst die Endung - $e$ für die erste Person Singular erworben.

- kommen: Die am häufigsten vorkommende Verbform ist komm $(-\varnothing)$. Auch für die 3. Pers. Pl. wird ausschließlich diese Verbform gebraucht (insgesamt fünfmal bei 4 Personen). Bei der 1. Pers. Sg. lässt sich die richtige Endung - $e$ bei drei Informantinnen finden. Bei POP, die sonst sehr wenig äußern konnte, handelt es sich eindeutig um die memorisierte Redewendung ich komme aus ..., die sie dreimal mit der richtigen Verbendung wiederholte. Bei ONG kommt ebenfalls nur eine einzige Verbform vor (komme), deshalb kann diese Endung nicht mit anderen Personen oder Pluralformen verglichen werden. A bildet eine

\footnotetext{
${ }^{14}$ Zwar ist die Verwendung des Flexivs - $\emptyset$ für die 1. Pers. Sg. in der gesprochenen Sprache gang und gäbe und wird häufig ebenfalls als korrekte Markierung betrachtet (vgl. z. B. Köpcke 1987), dennoch wird diese Form in der vorliegenden Analyse als falsch klassifiziert. Zum einen beeinträchtigt die Mitberücksichtigung dieser Form das Gesamtbild der richtig gebrauchten Flexionsmorpheme nicht, der Anteil der richtigen Verbformen mit - $\emptyset$ bleibt ohnehin hoch. Zum anderen gibt es Fälle, wie z. B. bei arbeiten, bei denen dieses Flexiv für die 1. Pers. Sg. nicht zulässig ist (vgl. etwa ich hab zwei kinder vs. *ich arbeit).
} 
ungewöhnliche Wortform kamme, was m. E. kein Präteritum ist, weil das Wort kurz gesprochen wird, sondern eine Kombination des englischen Verbs come mit der deutschen Flexionsendung $-e$, denn sie benutzt an anderen Stellen mehrheitlich das englische Verb come. Die Verbform mit -en kommt nur zweimal vor, als Infinitiv bei SOI und für die 3. Pers. Pl. bei OI. Im Allgemeinen scheinen die Informantinnen das Flexionsparadigma dieses Verbs noch nicht erworben zu haben. Der Anteil der richtig verwendeten Flexionsmorpheme ist noch niedrig. Nur wenn man komm bei der 3. Pers. Sg. wegen der Ausspracheschwierigkeiten bei Konsonantenclustern für richtig halten würde, würde sich die Anzahl richtiger Verbformen deutlich erhöhen. Diese Interpretation scheint allerdings bei diesem Verb nicht so plausibel zu sein, weil die Wortform komm überall vorkommt.

- sprechen: Das Verb kommt bei zwei Informantinnen (SOI und JOOB) im Partizip Perfekt vor, und zwar mit Ablaut und dem Flexionsmorphem für starke Verben als gesprochen. Als Infinitiv tritt es mehrheitlich mit der richtigen Endung -en auf. Allerdings gibt es bei den Infinitiven Abweichungen mit $-\varnothing$ oder $-e$. Auffällig bei diesem Verb ist das Phänomen, dass keine einzige Informantin bei den intendierten finiten Verbformen den Wechsel von $e$ zu $i$ im Verbstamm realisiert. Die Personalendungen für die 2. Pers. und 3. Pers. Sg., -st und - $t$, treten nicht auf. Es gibt zwar zwei Belege für die Endung $-s$ und für eine falsche Vokaländerung, aber es scheint sich eher um versprochene Wörter zu handeln (sprache für die 3. Pers. Sg. bei MAI und spreches für die 1. Pers. Pl. bei JOOB). Dieses Resultat bestätigt Parodis (2000: 373) Auffassung, dass sprechen zwar zu den häufig gebrauchten Verben gehört, aber keinen besonders hohen Grad der Subjekt-Verb-Kongruenz bei L2-Sprechern aufweist. Dass der richtige Gebrauch von Flexionsmorphemen zunächst bei infiniten Verbformen zu finden ist, vor allem bei Partizipien, wird bei diesem Verb noch einmal bestätigt, denn auch die Vokaländerung im Stamm wird bei diesem Verb nur in der Bildung des Partizips Perfekt realisiert.

\section{Gruppe}

- können: Auf den ersten Blick fragt man sich, ob die Informantinnen die Grundform dieses Verbs überhaupt kennen, wenn sie fast nur die Verbform kann gebrauchen, die lautlich anders als der Verbstamm realisiert wird. Bei BAS kommen könn und konn jeweils ein Mal vor, so dass es vorstellbar ist, dass sie dieses Verb auch in anderen Wortformen kennt. Allerdings handelt es sich hier um Fehler und sie produziert keine einzige richtige Verbform mit könn-. JOOB bildet einmal die intendierte Form von können mit der Endung -en, gebraucht allerdings den falschen Stamm, weil sie das englische Verb can mit der deutschen Flexionsendung kombiniert. Der Ablaut ist im Vergleich zu einer systematischen Markierung durch Flexionsmorpheme eindeutig einfacher zu erwerben. Durch die Vokaländerung ist ein neues Lexem entstanden, und die Informantinnen speichern die Wortform kann als ein dem englischen Wort can entsprechendes Lexem und benutzen es konsequent ohne Änderung bzw. ohne eine zusätzliche Endung hinzuzufügen. Anhand der vorliegenden Daten kann man noch nicht feststellen, ob das Verbparadigma für dieses Verb erworben wurde, oder nur die Verbform kann als eine lexikalische Einheit. Dazu kommt noch die Schwierigkeit, dass dieses im Deutschen häufig benutzte Verb zu den Chunks gehört und über den Erwerbsstand nicht viel aussagen kann, solange bei diesen Informantinnen keine Varianz der Verbformen desselben Verbs vorliegt.

- wissen: Bei diesem Verb ergibt sich ein ähnliches Bild. Die am häufigsten gebrauchte Verbform ist weiß $\beta$, bei fehlerhafter Aussprache als wei realisiert. Wie kann gehört diese Verbform zu den Chunks. Redewendungen wie ich weiß (nicht), ich kann (nicht) gebraucht man schon ganz früh, ohne das Flexionsparadigma gelernt oder erworben zu haben. Allerdings kann bei einer einzigen Informantin der Erwerb des Flexionsparadigmas von 
wissen festgestellt werden, auch wenn sich die Vollständigkeit noch nicht sichern lässt. Bei A treten neben wei $(\beta)$ auch wissen für die 3 . Pers. Sg. sowie als Infinitiv und gewuss als Partizip Perfekt auf. Die dominierende Form bleibt trotzdem wei $(\beta)$. Da die Informantin im Auslaut $-s t$ nie und $-s$ selten realisiert, ist es bei ihr ohnehin nicht möglich, die Verbform für die 2. Pers. Sg. weißt von weiß zu unterscheiden. Dass der Verbstamm auf [s] endet, erschwert den Erwerb der Flexion wie auch dessen Analyse. Die aufgetretenen Varianten des Verbs wissen bei A bestätigen allerdings die bis jetzt besprochene Annahme, dass zunächst infinite Verbformen erworben werden und die morphologische Markierung der finiten Verben erst spät erworben wird. Dabei wird eine morphologische Markierung zuerst mittels Vokalwechsel vorgenommen. Erst ganz zum Schluss erscheint eine Flexion mittels Personalendung.

Ungewöhnlicherweise wird die Endung - $e$ für die erste Person verwendet. Die Verbform weiße existiert im Paradigma dieses Verbs nicht. Dieser Fehler könnte m. E. auf drei mögliche Faktoren zurückgeführt werden. Erstens kann es sich um einen Sprossvokal handeln, den die Informantinnen hinzufügen, weil der Auslaut [s] für sie schwierig auszusprechen ist. Zweitens gilt $-e$ als übliche Endung für die erste Person Singular, deshalb wird sie übergeneralisiert. Drittens könnte es sein, dass die möglicherweise im Input häufig auftauchende Wendung ich weiß es (nicht), wenn die etwas schwierige Rolle von "es" noch nicht verstanden wird, als *ich weiße (nicht) "zurechtgehört" bzw. analysiert wird, was die Übergeneralisierung unterstützt. Alle drei Faktoren können darüber hinaus miteinander korrelieren bzw. einander begünstigen.

\section{Gruppe}

- haben: Bei diesem Verb treten fast alle richtigen Verbformen bei fast jeder Personalendung im Präsens (habe, hast, hat, haben) auf. Abweichungen kommen ebenfalls vor, allerdings erwartungsgemäß nur in geringem Umfang. Im Korpus tritt das Verb nie als Infinitiv oder Partizip auf. Auffällig ist die Erscheinung dieses Verbs im Präteritum, allerdings nur ein Mal bei einer Person (hatte bei JOOB). Bei diesem Verb lässt sich der Einfluss des Berndeutschen finden. Die Verbform ha für die erste Person tritt bei 3 Informantinnen auf.

- sein: Sieht man von der teilweise rudimentären Aussprache ab, muss man den Schluss ziehen, dass fast alle Verbformen von sein richtig gebraucht werden. Auch für dieses Verb lassen sich weder Infinitiv noch Partizip Perfekt finden. Die falschen Verbformen von sein bilden erwartungsgemäß nur einen geringen Anteil. Allerdings ist hier einiges anzumerken. Erstens tritt keine einzige Verbform mit der Endung - $(e) n$ auf (sein, waren). Nur die sogenannten Singular-Varianten liegen vor, und zwar mehrheitlich in der 1. und 3. Pers. Sing.: bin, war, is(t). Die Verbformen für die 2. Pers. Sg. liegen zwar vor, allerdings nur in minimaler Anzahl: wars (1 Mal bei BAS) und bist (1 Mal bei JOOB). Zweitens werden alle diese Formen, insbesondere war und is übergeneralisiert und auch für die 1. und vor allem 3. Pers. Pl. gebraucht. Nur ein Fehler war ungewöhnlich. NUCH sagt in einer Äußerung wir bin. Es kann entweder ein versprochenes Wort sein, weil sonst bei ihr bin konsequent nur für die 1. Pers. Sg. und is konsequent für die 3. Pers. Sg. gebraucht werden, oder aber auch ein übergeneralisierter Gebrauch der Singular-Varianten, ein ähnliches Phänomen wie bei war und is. Diese Daten zeigen, dass die Informantinnen die Pluralformen, wie etwa sind und waren, eindeutig noch nicht erworben haben. Es besteht die Tendenz, dass zwar das Kopulaverb mehrheitlich in richtigen Verbformen vorkommt, aber es handelt sich eher um memorisierte Wortformen. Der hohe Anteil an korrekten Verbformen ist kein Indikator dafür, dass die Informantinnen das Flexionsparadigma dieses Verbs erworben haben. Zumindest der vollständige Erwerb dieses Verbparadigmas muss bezweifelt werden. Nicht zuletzt ist bei diesem sicherlich am häufigsten gehörten und verwendeten Verb der Einfluss des 
Berndeutschen ebenfalls beobachtbar. Für die 3. Pers. Sg. liegt bei zwei Personen (NI, SO) die Form isch vor, die im Berndeutschen anstelle von ist benutzt wird.

Der Anteil der richtig gebrauchten Verbformen bei diesen 10 Verben wird in Tabelle 4 dargestellt. In jeder Gruppe werden die Verben entsprechend dem jeweiligen Anteil an richtig gebrauchten Verbformen aufgeführt. Die Verbformen (tokens) werden wiederum in die drei Gruppen Partizip Perfekt, Infinitiv und Finitum eingeteilt. Bei Kategorien, die mit keiner einzigen Verbform im Korpus belegt sind, wird nichts angegebenen und die Spalte wird schattiert, um sie von den Fällen zu unterscheiden, wo die intendierte Form vorhanden ist, aber nur eine falsche Form vorliegt $($ Anzahl =0). In der letzten Zeile wird der Anteil der Verbformen $-\varnothing$ bei der 3. Pers. Sg. als "richtig" mitberechnet und die Ergebnisse daraus als "eventuell richtig" dargestellt. Daraus ergibt sich, dass bei einigen Verben nun ein viel höherer Prozentanteil der richtigen Verbformen vorliegt. Dies soll zeigen, dass die Aussprache eine bedeutende Rolle spielt und große Auswirkungen auf den Erwerb der Flexion haben kann.

Tabelle 4: Anzahl und Anteil der richtig gebrauchten Verbformen bei den 10 untersuchten Verben

\begin{tabular}{|c|c|c|c|c|c|c|c|c|c|c|}
\hline & \multicolumn{3}{|c|}{ 1. Gruppe } & \multicolumn{3}{|c|}{ 2. Gruppe } & \multicolumn{2}{|c|}{ 3. Gruppe } & \multicolumn{2}{|c|}{ 4. Gruppe } \\
\hline & arbeiten & machen & sagen & kommen & sprechen & gehen & wissen & können & haben & sein \\
\hline $\begin{array}{l}\text { Part II } \\
\text { (r) }\end{array}$ & 0 & 19 & 100 & 0 & 2 & & 2 & & & \\
\hline Inf. (r) & 11 & 14 & 0 & 1 & 25 & 9 & 1 & & & \\
\hline fin. $(r)$ & 5 & 12 & 12 & 6 & 19 & 49 & 62 & 93 & 289 & 232 \\
\hline $\begin{array}{l}\text { Total } \\
(\mathrm{r})\end{array}$ & 14 & 45 & 112 & 7 & 46 & 58 & 63 & 93 & 289 & 232 \\
\hline $\begin{array}{l}\text { Anzahl } \\
\text { tokens } \\
\text { total }\end{array}$ & 81 & 119 & 151 & 50 & 103 & 100 & 106 & 107 & 347 & 240 \\
\hline$\%(r)$ & 19.44 & 27.82 & 74.17 & 16.00 & 44.66 & 58.00 & 59.43 & 86.92 & 82.29 & 96.67 \\
\hline $\begin{array}{l}\% \\
\text { evtl. (r) }\end{array}$ & & 58.82 & 81.46 & 50.00 & & 59.00 & 85.85 & & & \\
\hline
\end{tabular}

Die Ausgangshypothese, dass der Grad des Flexionserwerbs mit der Verbgruppe steigt (1. Gruppe am niedrigsten und 4. Gruppe am höchsten), kann damit zwar als bestätigt angesehen werden, aber nur, wenn man den Anteil der richtig gebrauchten Partizipien ausschließt. Im Allgemeinen beherrschen die Informantinnen in der vorliegenden Untersuchung die Flexion noch nicht. Auch der große Anteil der richtig gebrauchten Verbformen bei den Verben der 3. und 4. Gruppe zeigt die deutliche Tendenz, dass diese richtigen Formen eher als lexikalische Elemente erworben werden, die richtig verwendet werden, weil die Frequenz im Input sehr hoch ist. Deshalb lassen sich hier noch keine klaren Anzeichen für den Erwerb der Flexion als grammatische Funktionen erkennen.

\section{$5 \quad$ Fazit}

Die Ergebnisse der Analyse des Formenrepertoires sowie des Erwerbsverlaufs der Verbflexion zeigen, dass der Spracherwerbsprozess thailändischer Immigrantinnen in der Deutschschweiz ähnlich wie im Deutscherwerb der Arbeitsimmigranten im natürlichen 
Kontext verläuft (vgl. vor allem Köpcke 1987), obwohl ihr Hochdeutscherwerb, zumindest teilweise, gesteuert verläuft.

Vor allem der Einsatz der Personalendungen für regelmäßige Verben scheint für sie längerfristig problematisch zu sein, obwohl die Regel dafür schon früh im Unterricht vermittelt wird. Im Gegensatz dazu wird das Partizip Perfekt gleich nach seiner Erscheinung mehrheitlich richtig gebildet, auch wenn solche Wortformen erst später entwickelt wurden. Ein weiterer Hinweis, dass der Unterricht eine untergeordnete Rolle spielt, ist die Tatsache, dass sich keine Übergeneralisierung der Partizipbildung für regelmäßige Verben mit $g e-+-t$ finden lässt, was im gesteuerten Unterricht neben der Form ge- + Infinitiv oder $g e-+$ Infinitiv-ähnliche Formen üblich ist (vgl. etwa Diehl et al. 2000: 149). Die Informantinnen schreiben den unterschiedlichen Wortformen eines Verbs zunächst vermutlich keine Funktion $\mathrm{zu}$, sondern verwenden Verbformen undifferenziert als unanalysierte Formen bzw. als lexikalische Einheiten.

Zwischen dem Erwerb der Verbflexion und dem Erwerb der Phonologie besteht eindeutig ein Zusammenhang. Vor allem weil die thailändischen Informantinnen Schwierigkeiten haben, die Auslautkonsonanten sowie -konsonantencluster auszusprechen, ist es umso schwieriger für sie, einige der Personalendungen richtig wiederzugeben. Die Ausspracheschwierigkeiten im Bereich der Konsonanten erschweren die Feststellung erworbener und nicht-erworbener Flexive bei diesen Informantinnen und wohl auch den Erwerb der Verbflexion an sich. Die Unfähigkeit, einige Auslautkonsonanten zu realisieren, könnten m. E. die Weiterentwicklung der morphologischen Markierung einigermaßen erschwert haben, zumindest wenn es sich um mündliche Kompetenz handelt. Dieses Phänomen ist vor allem bei zwei Informantinnen, SOI und A, auffällig. Dass bei ihnen die Personalendung $-s t$ oder die abweichende Form $-s$ nie vorkommt und stattdessen der Auslaut - $t$ auftritt, führt zu der Schlussfolgerung, dass ihr Deutscherwerb im Hinblick auf die Aussprache dieser Auslaute fossiliert ist. D. h. es ist denkbar, dass die beiden das Flexionsmorphem -st zur Markierung der 2. Pers. Sg. im Deutschen nie erwerben oder zumindest nie artikulieren.

Die Ausgangssprache hat somit zwar keine auffälligen Auswirkungen auf die Erwerbsfolge der verbalen Flexionsmorpheme, vor allem bei Personalendungen, aber auf die lautliche Differenziertheit flexionsmorphologischer Kategorien, was einen langsamen Erwerbsprozess verursachen kann. Besonders der Ausbau der Konsonanten und der Konsonantencluster am Silbenrand, der für die Flexionsmorpheme charakteristisch ist, bereitet den thailändischen Immigrantinnen große Schwierigkeiten beim Erwerb der Verbflexion. Dadurch ist vor allem die Übergeneralisierung der Form - $\varnothing$ schwer zu bewältigen. Es ist aber durchaus möglich, dass die Informantinnen über das Wissen verfügen, welche Endungen sie eigentlich realisieren müssten, dass sie dieses Wissen aber wegen mangelnder Sprech- bzw. Artikulationskompetenz nicht umsetzen können. Als nächster Schritt wäre deshalb zu überprüfen, ob sie die Flexionsmorpheme zumindest in ihrer kognitiven Wahrnehmung erworben haben, indem beispielsweise auch ihre schriftlichen Äußerungen oder ihre explizite Grammatikkenntnis mit einbezogen werden. Nicht weniger wichtig ist es sicherlich auch, den Zusammenhang zwischen dem Erwerb der Verbflexion und anderen Aspekten zu erforschen. Es wäre beispielsweise interessant zu untersuchen, ob die syntaktischen Mittel wie z. B. Verbstellung für diese Informantinnen einfacher zu erwerben wäre, oder ob sie deutlich mehr lexikalische Einheiten zu Hilfe nehmen, um die Funktion der Flexionsmorpheme der Verben zu ersetzen, wenn die Morphologie für sie schwer erlern- bzw. umsetzbar ist. 


\section{Literaturangaben}

Attaviriyanupap, Korakoch (2004): "Wie 'Haar' zu 'Maus' wird: Geschlechtsbezogene pronominale Referenz im Thailändischen". Linguistik online 21, 4/04: 3-22. http://www.linguistik-online.de/21_04/kora.html.

Attaviriyanupap, Korakoch (2006): "Ausspracheabweichungen im Hochdeutsch thailändischer Immigrantinnen in der Deutschschweiz". Linguistik online 26, 1/06: 15-30. http://www.linguistik-online.de/26_06/attaviriyanupap.html.

Blackshire-Belay, Carol (1991): Language Contact. Verb Morphology in German of Foreign Workers. Tübingen. (= Tübinger Beiträge zur Linguistik; 356).

Diehl, Erika et al. (2000): Grammatikunterrricht: Alles für der Katz?. Untersuchungen zum Zweitsprachenerwerb Deutsch. Tübingen. (= Reihe Germanistische Linguistik 220).

Dietrich, Rainer/Klein, Wolfgang/Noyau, Colette (1995): The acquisition of temporality in a second language. Amsterdam/Philadelphia. (= Studies in Biliangualism Vol. 7).

Helbig, Gerhard/Buscha, Joachim (2001): Deutsche Grammatik. Ein Handbuch für den Ausländerunterricht. Bern etc.

Hentschel, Elke/Weydt, Harald (2003): Handbuch der deutschen Grammatik. 3., völlig neu bearb. Aufl. Berlin/New York.

Köpcke, Klaus-Michael (1987): "Der Erwerb morphologischer Ausdrucksmittel durch L2Lerner am Beispiel der Personalflexion". Zeitschrift für Sprachwissenschaft 6,2 (1987): 186-205.

Maurer, Ernst (2001): Deutsch in der Schweiz. Zug.

Parodi, Teresa (2000): "Finiteness and verb placement in second language acquisition". Second Language Research 16, 4: 355-381.

Ruoff, Arno (1981): Häufigkeitswörterbuch gesprochener Sprache. Tübingen. (= Veröffentlichung der Tübinger Arbeitsstelle Band 8).

von Stutterheim, Christine (1986): Temporalität in der Zweitsprache. Eine Untersuchung zum Erwerb des Deutschen durch türkische Gastarbeiter. Berling/New York.

Tmangraksat, Pornsan (1985): Der Erwerb der deutschen Tempusflexion bei thailändischen Erwachsenen. Frankfurt a. M. etc. (= Europäische Hochschulschriften: Reihe 21 Linguistik; Bd. 42). 